\title{
Near-Inertial Waves and Turbulence Driven by the Growth of Swell
}

\author{
Gregory L. Wagner, ${ }^{a}$ Gregory P. Chini, ${ }^{b}$ Ali RAmadhan, ${ }^{a}$ Basile GAllet, ${ }^{c}$ And RafFAele Ferrari ${ }^{\mathrm{a}}$ \\ ${ }^{\mathrm{a}}$ Earth, Atmospheric, and Planetary Sciences, Massachusetts Institute of Technology, Cambridge, Massachusetts \\ ${ }^{\mathrm{b}}$ Integrated Applied Mathematics and Mechanical Engineering, University of New Hampshire, Durham, New Hampshire \\ ' Service de Physique de l'Etat Condense, Commissariat á l'Energie Atomique Saclay, CNRS UMR 3680, Universitè Paris-Saclay, \\ Saint-Aubin, France
}

(Manuscript received 30 July 2020, in final form 18 December 2020)

\begin{abstract}
Between 5\% and 25\% of the total momentum transferred between the atmosphere and ocean is transmitted via the growth of long surface gravity waves called "swell." In this paper, we use large-eddy simulations to show that swelltransmitted momentum excites near-inertial waves and drives turbulent mixing that deepens a rotating, stratified, turbulent ocean surface boundary layer. We find that swell-transmitted currents are less effective at producing turbulence and mixing the boundary layer than currents driven by an effective surface stress. Overall, however, the differences between swelldriven and surface-stress-driven boundary layers are relatively minor. In consequence, our results corroborate assumptions made in Earth system models that neglect the vertical structure of swell-transmitted momentum fluxes and instead parameterize all air-sea momentum transfer processes with an effective surface stress.
\end{abstract}

KEYWORDS: Ocean; Turbulence; Waves, oceanic; Wind stress; Boundary layer; Large eddy simulations

\section{Introduction}

Winds flowing over the ocean excite a spectrum of surface gravity waves ranging from ripples to kilometer-long swells. Beneath persistent winds, the statistics of the shortest gravity waves saturate in a stationary balance between wind input, nonlinear interactions between wave components, and dissipation and breaking (Phillips 1985). The saturated fraction of the wave spectrum is called the "equilibrium range." Longer waves, or "swell," are out of equilibrium by definition and therefore grow along the fetch of the wind.

Much of the momentum input into the ocean is transferred via form stress acting on the faces of equilibrium range waves (Grare et al. 2013; Melville 1996). The shortness of equilibrium range waves and the accompanying effects of wave breaking motivate the parameterization of air-sea momentum transfer as an effective stress imposed at the air-sea interface. Yet some fraction of the total momentum transferred between the atmosphere and ocean-perhaps as small as 5\% in conditions typical to the laboratory and field experiments (Melville 1996), or as large as $25 \%$ beneath hurricane-strength winds (Fan et al. 2009) - is not transferred to the ocean at or just beneath the surface, but is instead distributed in depth by pressure gradients associated with the growth of swell. In this paper, we address the effects of the "swell-mediated" fraction of the total momentum flux on turbulent ocean surface boundary layers.

Swell mediates momentum transfer between the atmosphere and ocean when swell is resonantly excited by coherent viscous stresses (Longuet-Higgins 1969) or a coherent component of the atmospheric pressure spectrum at the sea surface. We illustrate this process by considering the oceanic response to the traveling atmospheric pressure disturbance

Corresponding author: Gregory L. Wagner, wagner.greg@ gmail.com

$$
p_{a}(x, t)=\rho_{0} P \cos (k x-\underbrace{\sqrt{g k}}_{\substack{\text { def } \\=}} t),
$$

where $\rho_{0}$ is an ocean reference density, $P$ is the ocean-side kinematic pressure, and $g$ is gravitational acceleration. Over an ocean at rest, the pressure field in (1) excites an infinite, monochromatic surface wave field with wavenumber $k$ and frequency $\sigma=\sqrt{g k}$ with surface displacement

$$
s(x, t)=\underbrace{\frac{P k}{2 \sigma}}_{\substack{\text { def } \\=} a(t)} t \sin (k x-\sigma t) .
$$

In (2) we define the time-dependent swell amplitude $a(t)$. If $p_{a}$ vanishes after some time $t=T$, the outcome is an infinite, steadily propagating wave field with amplitude $a(T)$. This outcome was first investigated in a seminal paper by Stokes (1847).

The depth-integrated, swell-averaged $x$-momentum budget for a nonrotating and inviscid ocean reveals the air-sea momentum transfer generated by (1),

$$
\partial_{t}\left\langle\int_{-h}^{s} u d z\right\rangle=\frac{1}{\rho_{0}}\left\langle p_{a} \partial_{x} s\right\rangle,
$$

where $h$ is a depth at which momentum fluxes vanish, and the angle brackets $\langle\cdot\rangle$ denote a simultaneous average over horizontal directions and a time average over the rapid oscillations of swell. The time derivative in (3) is the rate of change of depth-integrated momentum over time scales much longer than the oscillation of the surface wave field.

The term $\left\langle p_{a} \partial_{x} s\right\rangle$ on the right of (3) describes atmospheric pressure forces impacting the tilted ocean surface and is called "form stress." With the pressure field in (1) and surface displacement in (2), we find that momentum is transferred to the ocean by form stress at the rate 


$$
\frac{1}{\rho_{0}}\left\langle p_{a} \partial_{x} s\right\rangle=\frac{(P k)^{2}}{4 \sigma} t
$$

A remarkable aspect of this scenario, first described by Stokes (1847), is that the form stress in (4) forces an irrotational current whose average velocity at a fixed position-the Eulerianmean velocity $\langle u\rangle$-is zero. In other words, (4) acting on a nonrotating ocean forces a current with $\langle u\rangle=0$, and

$$
\begin{aligned}
\partial_{t}\left\langle\int_{-h}^{s} u d z\right\rangle & =\partial_{t} \int_{-h}^{0} u^{S} d z=\frac{(P k)^{2}}{4 \sigma} t, \text { where } \\
u^{S}(z, t) & =e^{2 k z} a(t)^{2} \sigma k,
\end{aligned}
$$

is called the "Stokes drift" (Bühler 2014). The rate of change of the depth-integrated Stokes drift (5) is equal to the air-sea momentum transfer due to form stress in (4).

In this paper, we numerically simulate the response of a rotating, stratified, turbulent boundary layer to the resonant atmospheric pressure forcing (1), as well as mixtures of effective surface stress and swell-mediated form stress with similar total momentum input. In these more general scenarios, the Eulerian-mean velocity is nonzero. However, the rate of change of the Stokes drift in (5) still measures the part of the air-sea momentum transfer rate associated with swell-coherent form stress. To form the total momentum budget, we define the complexified total boundary layer momentum and depthintegrated Stokes drift,

$U^{L}(t) \stackrel{\text { def }}{=}\left\langle\int_{-h}^{s} u+i v d z\right\rangle$, and $U^{S}(t) \stackrel{\operatorname{def}}{=} \int_{-h}^{0} u^{S}+i v^{S} d z$

and parameterize the net air-sea momentum transfer rate with $\tau+\partial_{t} U^{S}$, where $\tau$ is a complexified effective surface stress that models the net effect of momentum transfer through swell-incoherent viscous stresses, form stress acting on equilibrium range waves, and the concomitant effects of wave breaking. The superscript " $L$ " in (6) stands for "Lagrangian-mean," whose agency is discussed in section 2. The governing equation for the total momentum $U^{L}$, which may be derived either from the rotating Navier-Stokes equations beneath a free surface, or from the CraikLeibovich wave-averaged Boussinesq equations (9)-(11) that form the basis for the simulations in this paper (Kukulka et al. 2010), is

$$
\partial_{t} U^{L}+i f U^{L}=\tau+\partial_{t} U^{S},
$$

where $f$ is the Coriolis parameter.

Ursell and Deacon (1950), Hasselmann (1970), Pollard (1970), and Eq. (7) show that, in the presence of rotation, the current that arises beneath a growing monochromatic wave field is not steady as in Stokes's (1847) problem, but instead rotates inertially. A surprising result of our numerical simulations is that, due to three-dimensionality, stratification, and preexisting turbulence, swell-transmitted flows both rotate inertially and transfer energy to turbulence via shear production, thus mixing and deepening the boundary layer. This is in striking contrast to the laminar, unidirectional flow that arises in the irrotational case analyzed by Stokes (1847) and exemplified by (5). The evolution of a turbulent boundary layer in our most basic case beneath a growing surface wave field with $\tau=0$ is depicted in Fig. 1. Setting $\tau=0$ distinguishes our work from Kukulka et al. (2010), Sullivan et al. (2012), and Large et al. (2019) that investigate time-dependent mixing processes due to a combination of time-dependent $\tau$ and $\partial_{t} U^{S}$, and which do not separately investigate the effects of the "additional" momentum input by nonzero $\partial_{t} U^{S}$.

Section 2 introduces the wave-averaged Boussinesq equations and discusses some of their basic properties. In section 3 , we continue beyond the scenario depicted in Fig. 1 to investigate the qualitative differences between the mixing and deepening of turbulent boundary layers forced either by an effective surface stress $\tau$ or swell growth via $\partial_{t} U^{S}$. We conclude that depth-distributed forcing by $\partial_{t} U^{S}$ produces less mixing than forcing by $\tau$, because $\partial_{t} U^{S}$ drives currents with weaker shear that relinquish less energy to turbulence. In shallow boundary layers, moreover, some of the swelltransmitted atmospheric forcing acts directly on laminar near-inertial motions below the base of the boundary layer. We emphasize that these observations pertain to the interior effects of boundary layer forcing mechanisms associated with the shear production of turbulence, rather than the surfaceconcentrated effects of wave breaking, which are neglected in our large-eddy simulations.

Langmuir turbulence is not the main focus of this work. Nevertheless, the wave-catalyzed organization of turbulent motions into the coherent structures of Langmuir turbulence (Sullivan and McWilliams 2010; D'Asaro et al. 2014) features prominently in our numerical solutions. In section $3 \mathrm{e}$, we observe that the coherent Langmuir turbulence structures tend to align with the Lagrangian-mean shear, consistent with Sullivan et al.'s (2012) results beneath realistic hurricane winds and waves. After the surface stress dies down, however, the orientation of the coherent structures decouples from the weakening Lagrangian-mean shear and locks onto $\hat{\mathbf{y}}$, the axis of the effective background vorticity associated with the $\hat{\mathbf{x}}$-propagating swell. We suggest an explanation for this phenomenon in the context of the Lagrangian-mean formulation of the wave-averaged Boussinesq equations.

Section 4 investigates the importance of swell growth rate in determining initial conditions for large-eddy simulations, and subsequent deepening of turbulent boundary layers under steady surface stress and steady surface waves. Section 5 concludes by discussing how our results may corroborate, a posteriori, some assumptions that underpin the parameterization of atmosphere-ocean momentum transfer in general circulation models. Appendix A describes the large-eddy simulation software "Oceananigans.jl," appendix B provides the vector calculus identities required to manipulate the Eulerian-mean Craik-Leibovich equations into their Lagrangian-mean form, and Table 1 lists the largeeddy simulations used in this work.

\section{The surface-wave-averaged Boussinesq equations}

The Lagrangian-mean velocity beneath surface gravity waves is 

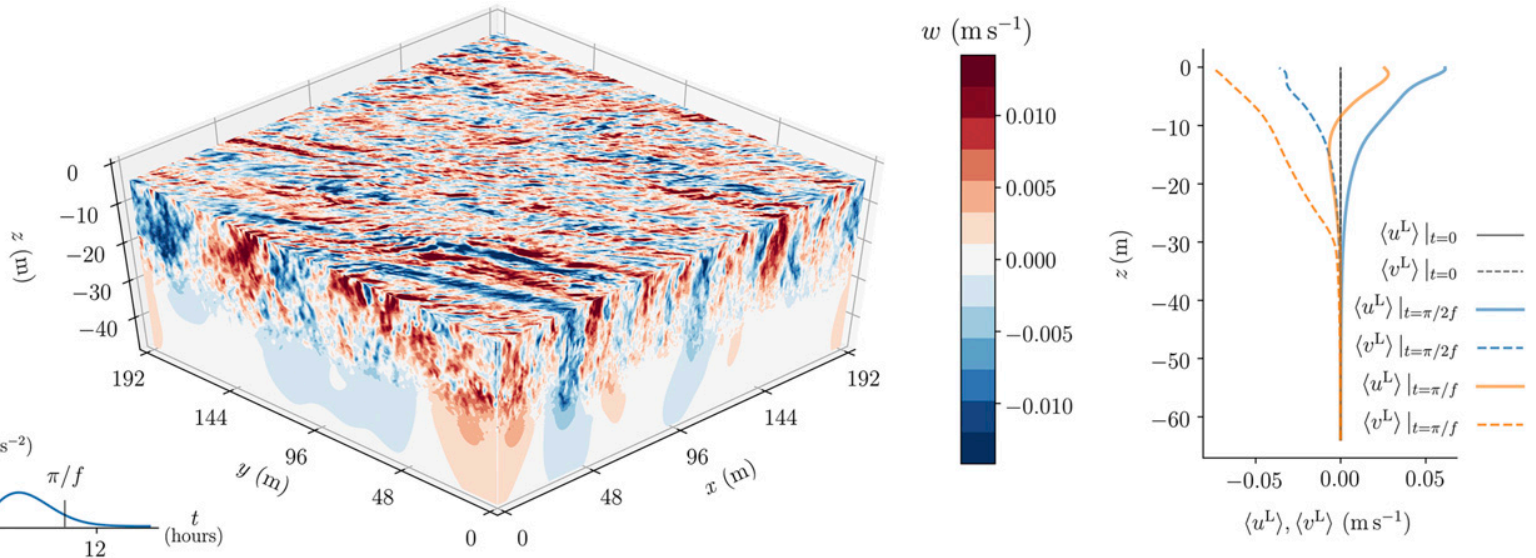

FIG. 1. Currents and turbulence beneath forced surface waves with the time-dependent Stokes drift $\mathbf{u}^{S}=e^{2 k z} a^{2} k \sqrt{g k}\left(1-e^{-t^{2} / 2 T_{w}}\right) \hat{\mathbf{x}}$ with wavenumber $k=2 \pi / 100 \mathrm{~m}^{-1}$, growth time scale $T_{w}=4 \mathrm{~h}$, equilibrium amplitude $a=2 \mathrm{~m}$, and gravitational acceleration $g=9.81 \mathrm{~m} \mathrm{~s}{ }^{-2}$. (left) Contours of $w^{L}$ and (right) the horizontally averaged horizontal velocity components $\left\langle u^{L}\right\rangle$ and $\left\langle v^{L}\right\rangle$ are shown after half an inertial period at $t=\pi / f$. The tendency of the depth integral of the Stokes drift, $\partial_{t} U^{S}=\int_{-h}^{0} u^{S} d z$, is a stress accompanying the growth of swell that forces a vertically sheared inertial oscillation and drives turbulent mixing and boundary layer deepening. More details about the physics, numerics, and software can be found in sections 2 and 3, in Table 1, and at https://github.com/glwagner/ WaveTransmittedTurbulence.jl.

$$
\mathbf{u}^{L} \stackrel{\text { def }}{=} \mathbf{u}^{E}+\underbrace{\overline{(\tilde{\boldsymbol{\xi}} \cdot \nabla) \tilde{\mathbf{u}}}}_{\stackrel{\text { def }}{=} \mathbf{u}^{S}},
$$

where $\tilde{\mathbf{u}}$ is oscillatory velocity associated with waves, $\tilde{\boldsymbol{\xi}}=(\tilde{\xi}, \tilde{\eta}, \tilde{\zeta})$ is the wavy zero-mean particle displacement defined via $\partial_{t} \tilde{\boldsymbol{\xi}}=\tilde{\mathbf{u}}$, the overline $\overline{()}$ is a running Eulerian time average-a "wave average"- over surface wave oscillations, $\mathbf{u}^{E} \stackrel{\text { def }}{=} \overline{\mathbf{u}}$ is the Eulerian-mean velocity, and $\mathbf{u}^{S}$ is the Stokes drift. The
Lagrangian-mean velocity $\mathbf{u}^{L}(x, y, z, t)$ advects mass, momentum, and vorticity, and its variance $(1 / 2)\left|\mathbf{u}^{L}\right|^{2}$ is the wave-averaged kinetic energy.

The wave-averaged Boussinesq equations in an $f$-plane tangent to and rotating with the ocean surface and cast in terms of $\mathbf{u}^{L}$ are (Craik and Leibovich 1976; Huang 1979; Leibovich 1980; Holm 1996; Suzuki and Fox-Kemper 2016; Seshasayanan and Gallet 2019)

TABLE 1. Simulations $1-8$ are reported in section 3 and simulations A-E are reported in section 4 . Simulations $1-5$ and A-E use $128 \mathrm{~m} \times$ $128 \mathrm{~m} \times 64 \mathrm{~m}$ domains with $256 \times 256 \times 256$ grid points and uniform $0.5 \mathrm{~m} \times 0.5 \mathrm{~m} \times 0.25 \mathrm{~m}$ grid spacing in $x, y, z$. Simulations $6-8$ use $192 \mathrm{~m} \times 192 \mathrm{~m} \times 96 \mathrm{~m}$ domains with $256 \times 256 \times 384$ grid points and uniform $0.75 \mathrm{~m} \times 0.75 \mathrm{~m} \times 0.25 \mathrm{~m}$ grid spacing in $x, y, z$. The effective wave-forced stress during the growth of 100-m wavelength, 1-m amplitude deep water waves on a time scale of $T_{w}=4 \mathrm{~h}$ is $\tau_{w}=2.72 \times$ $10^{-5} \mathrm{~m}^{2} \mathrm{~s}^{-2}$. The wind stress prescribed by McWilliams et al. (1997) is $\tau_{\mathrm{MSM}}=-3.72 \times 10^{-5} \mathrm{~m}^{2} \mathrm{~s}^{-2}$. Simulation 1 is initialized with $\mathbf{u}^{L}=10^{-6} \times e^{z / 2} \chi_{u}(x, y, z)\left(\left.\Delta_{z} q_{z}\right|_{t=0}\right)^{1 / 3}$ and $b=N^{2} z+10^{-6} \times e^{z / 2} \chi_{b}(x, y, z) N^{2} \Delta_{z}$, where each $\chi_{\psi}$ is a Gaussian-distributed, unit standard deviation random field for each $\psi \in\left(u^{L}, v^{L}, w^{L}, b\right), \Delta_{z}=0.25 \mathrm{~m}$ is the vertical grid spacing, and $N^{2}=10^{-6} \mathrm{~s}^{-2}$ is the initial buoyancy gradient. Simulations 2-7 are initialized from simulation 1 at $t=\pi / f$ with Coriolis parameter $f=10^{-4} \mathrm{~s}^{-1}$. Simulations A-E use $u_{0}^{S}=0.068 \mathrm{~m} \mathrm{~s}^{-1}$ and $k=0.105 \mathrm{~m}^{-1}$ The simulation data were generated with Oceananigans (https://github.com/CliMA/Oceananigans.jl). Instructions for reproducing the results are at https://github.com/glwagner/WaveTransmittedTurbulence.jl.

\begin{tabular}{lllccc}
\hline \hline ID & \multicolumn{1}{c}{ Simulation name } & \multicolumn{1}{c}{ Stokes drift } & Surface conditions & Domain size & Grid spacing \\
\hline 1 & Weak spinup & $u^{S}=0$ & $q_{\mathrm{z}}=5 \times 10^{-10} \mathrm{~m}^{2} \mathrm{~s}^{-3}$ & Regular & $0.5 \mathrm{~m} \times 0.5 \mathrm{~m} \times 0.25 \mathrm{~m}$ \\
2 & Weak growing waves & $u^{S}=u_{\mathrm{eq}}^{S}(z)\left(1-e^{-t^{2} / 2 T_{w}^{2}}\right)$ & Free slip, insulating & Regular & $0.5 \mathrm{~m} \times 0.5 \mathrm{~m} \times 0.25 \mathrm{~m}$ \\
3 & Weak surface stress, no waves & $u^{S}=0$ & $\mathcal{T}_{x z}=-\tau_{w} e^{-t^{2} / 2 T_{w}^{2}} t / T_{w}$ & Regular & $0.5 \mathrm{~m} \times 0.5 \mathrm{~m} \times 0.25 \mathrm{~m}$ \\
4 & Weak surface stress, steady waves & $u^{S}=u_{\mathrm{eq}}^{S}(z)$ & $\mathcal{T}_{x z}=-\tau_{w} e^{-t^{2} / 2 T_{w}^{2} t / T_{w}}$ & Regular & $0.5 \mathrm{~m} \times 0.5 \mathrm{~m} \times 0.25 \mathrm{~m}$ \\
5 & Weak surface stress, growing waves & $u^{S}=u_{\mathrm{eq}}^{S}(z)\left(1-e^{-t^{2} / 2 T_{w}^{2}}\right)$ & $\mathcal{T}_{x z}=-\tau_{w} e^{-t^{2} / 2 T_{w}^{2}} t / T_{w}$ & Regular & $0.5 \mathrm{~m} \times 0.5 \mathrm{~m} \times 0.25 \mathrm{~m}$ \\
6 & Strong spinup & $u^{S}=0$ & $q_{z}=5 \times 10^{-10} \mathrm{~m}^{2} \mathrm{~s}^{-3}$ & Large & $0.75 \mathrm{~m} \times 0.75 \mathrm{~m} \times 0.25 \mathrm{~m}$ \\
7 & Strong growing waves & $u^{S}=4 u_{\mathrm{eq}}^{S}\left(1-e^{-t^{2} / 2 T_{w}^{2}}\right)$ & Free slip, insulating & Large & $0.75 \mathrm{~m} \times 0.75 \mathrm{~m} \times 0.25 \mathrm{~m}$ \\
8 & Strong surface stress, no waves & $u^{S}=0$ & $\mathcal{T}_{x z}=-4 \tau_{w} e^{-t^{2} / 2 T_{w}^{2} t / T_{w}}$ & Large & $0.75 \times 0.75 \times 0.25 \mathrm{~m}$ \\
9 & Strong surface stress, steady waves & $u^{S}=4 u_{\mathrm{eq}}^{S}(z)$ & $\mathcal{T}_{x z}=-4 \tau_{w} e^{-t^{2} / 2 T_{w}^{2} t / T_{w}}$ & Large & $0.75 \mathrm{~m} \times 0.75 \mathrm{~m} \times 0.25 \mathrm{~m}$ \\
A & Reference & $u^{S}=0$ & $\mathcal{T}_{x z}=\tau_{\mathrm{MSM}}$ & Regular & $0.5 \times 0.5 \times 0.25 \mathrm{~m}$ \\
B & 1× excited & $u^{S}=e^{2 k z} u_{0}^{S}$ & $\mathcal{T}_{x z}=\tau_{\mathrm{MSM}}$ & Regular & $0.5 \mathrm{~m} \times 0.5 \mathrm{~m} \times 0.25 \mathrm{~m}$ \\
$\mathrm{C}$ & $1 \times$ resting & $u^{S}=e^{2 k z} u_{0}^{S}$ & $\mathcal{T}_{x z}=\tau_{\mathrm{MSM}}$ & Regular & $0.5 \mathrm{~m} \times 0.5 \mathrm{~m} \times 0.25 \mathrm{~m}$ \\
$\mathrm{D}$ & $4 \times$ excited & $u^{S}=e^{2 k z} 4 u_{0}^{S k z} 4 u_{0}^{S}$ & $\mathcal{T}_{x z}=\tau_{\mathrm{MSM}}$ & Regular & $0.5 \mathrm{~m} \times 0.5 \mathrm{~m} \times 0.25 \mathrm{~m}$ \\
$\mathrm{E}$ & $4 \times$ resting & $\mathcal{T}_{x z}=\tau_{\mathrm{MSM}}$ & Regular & $0.5 \mathrm{~m} \times 0.5 \mathrm{~m} \times 0.25 \mathrm{~m}$ \\
\hline
\end{tabular}




$$
\begin{gathered}
\partial_{t} \mathbf{u}^{L}+\left(\mathbf{u}^{L} \cdot \nabla\right) \mathbf{u}^{L}+\left(f \hat{\mathbf{z}}-\nabla \times \mathbf{u}^{S}\right) \times \mathbf{u}^{L}+\nabla p \\
=b \hat{\mathbf{z}}-\nabla \cdot \mathcal{T}+\partial_{t} \mathbf{u}^{S} \\
\partial_{t} b+\mathbf{u}^{L} \cdot \nabla b=-\nabla \cdot \mathbf{q}, \\
\nabla \cdot \mathbf{u}^{L}=0,
\end{gathered}
$$

where $p$ is Eulerian-mean kinematic pressure, $b$ is Eulerianmean buoyancy, $f$ is the Coriolis parameter, and $\mathcal{T}$ and $\mathbf{q}$ are the stress tensor and buoyancy flux due either to molecular diffusion or a subfilter turbulent diffusion model for large-eddy simulation. We show how (9)-(11) are derived from the Eulerian-mean form of the Craik-Leibovich equations, and how they are connected to the generalized Lagrangian-mean equations derived by Andrews and McIntyre (1978) in appendix B.

Equations (9)-(11) are an asymptotic approximation of the Navier-Stokes equations beneath a small amplitude and weakly modulated surface wave field. In particular, (11) neglects a term relevant for time-dependent swell and associated with divergence of the vertical component of the Stokes drift. If accounted for, this vertical divergence would lift the mean position of each fluid parcel by $(1 / 2) a^{2} k e^{2 k z}$ as the swell amplitude $a$ increases [see Mcintyre (1981) and Eq. (3.7) in Longuet-Higgins (1986)]. In the cases considered in this paper, however, the vertical velocity associated with this mean vertical displacement is miniscule: for example, the growth of $a=2 \mathrm{~m}$ amplitude swell with wavenumber $k=2 \pi / 100 \mathrm{~m}^{-1}$ lifts the sea surface by just $(1 / 2) a^{2} k=0.12 \mathrm{~m}$ over a period of $4 \mathrm{~h}$. Because the effect is so small, we neglect the vertical component of the Stokes drift and prescribe $w^{L}=0$ at $z=0$.

The momentum equation (9) is often written as a prognostic equation for the Eulerian-mean velocity $\mathbf{u}^{E}$, both for analysis (Craik and Leibovich 1976; Suzuki and Fox-Kemper 2016) and large-eddy simulation (Skyllingstad and Denbo 1995; McWilliams et al. 1997; Noh et al. 2004; Polton and Belcher 2007; Harcourt and D'Asaro 2008; Yang et al. 2015). We use the Lagrangian-mean velocity $\mathbf{u}^{L}$ instead as our prognostic variable for both numerical simulations and analysis. Using $\mathbf{u}^{L}$ explicitly identifies the role of atmospheric momentum forcing transmitted via growing swell, and thus $\partial_{t} \mathbf{u}^{S}$, in driving the turbulent evolution of the simulated surface boundary layers in section 3.

Prescribing $\mathbf{u}^{S}(\mathbf{x}, t)$ in (9) determines the effects of swell on the evolution of the Lagrangian-mean momentum $\mathbf{u}^{L}$. Vertical fluxes of horizontal momentum into $\mathbf{u}^{L}$ through saturated surface waves in the equilibrium range are prescribed through stress boundary conditions on $\mathcal{T}_{x z}$ and $\mathcal{T}_{y z}$ at $z=0$. Buoyancy fluxes are prescribed through $\left.q_{z}\right|_{z=0}$. The complexified downward surface stress in (7) is $\tau \stackrel{\text { def }}{=}-\mathcal{T}_{x z}-\left.i \mathcal{T}_{y z}\right|_{z=0}$.

\section{a. Wave-averaged effective background vorticity}

The term

$$
\underbrace{\left(f \hat{\mathbf{z}}-\nabla \times \mathbf{u}^{S}\right)}_{\stackrel{\text { def }}{=} \mathbf{\Omega}^{\dagger}} \times \mathbf{u}^{L}
$$

appears in the momentum equation (9), where $f \hat{\mathbf{z}}$ is the planetary vorticity and $\nabla \times \mathbf{u}^{S}$ is the surface wave pseudovorticity.
As discussed by Bühler (2014) in their section 11.3.2, (12) means that the effective background vorticity $\Omega^{\dagger}$ is advected by the Lagrangian-mean velocity $\mathbf{u}^{L}$. In other words, the total vorticity is

$$
\boldsymbol{\Omega} \stackrel{\text { def }}{=} \boldsymbol{\Omega}^{\dagger}+\nabla \times \mathbf{u}^{L}=f \hat{\mathbf{z}}+\nabla \times \underbrace{\left(\mathbf{u}^{L}-\mathbf{u}^{S}\right)}_{=\mathbf{u}^{E}},
$$

in the sense that $\nabla \times(9)$ with $b=0$ and $\mathcal{T}=0$ is

$$
\partial_{t} \mathbf{\Omega}+\left(\mathbf{u}^{L} \cdot \nabla\right) \boldsymbol{\Omega}=(\boldsymbol{\Omega} \cdot \nabla) \mathbf{u}^{L} .
$$

Equation (14) resembles the usual vorticity equation for a rotating fluid, except that $\boldsymbol{\Omega}$ stands for vorticity rather than $f \hat{\mathbf{z}}+\nabla \times \mathbf{u}^{L}$. While $\mathbf{u}^{L}$ transports mass, momentum, and vorticity, the wave-averaged deviation from planetary vorticity is $\nabla \times \mathbf{u}^{E}$, rather than $\nabla \times \mathbf{u}^{L}$. This altered relationship between momentum and vorticity encapsulates the dynamical effect of surface waves on the evolution of $\mathbf{u}^{L}$. We use the interpretation of $\boldsymbol{\Omega}^{\dagger}$ as an effective background vorticity to explain some of the phenomena observed in our large-eddy simulations in section 5 .

\section{b. Wave-averaged kinetic energy}

The conservation law for volume-integrated wave-averaged kinetic energy, (1/2) $\left|\mathbf{u}^{L}\right|^{2}$, in unstratified flow with $b=0$ and inviscid flow with $\mathcal{T}=0$ follows from $\int \mathbf{u}^{L} \cdot(9) d V$,

$$
\frac{d}{d t} \int \frac{1}{2}\left|\mathbf{u}^{L}\right|^{2} d V=\int \mathbf{u}^{L} \cdot \partial_{t} \mathbf{u}^{S} d V,
$$

where we have assumed there are no momentum fluxes across the boundary of $V$. The total mean kinetic energy $\int(1 / 2)\left|\mathbf{u}^{L}\right|^{2} d V$ is therefore conserved beneath steady surface waves in unstratified, inviscid flow: there is no energy exchange between $\mathbf{u}^{L}$ and steady surface waves. Equation (15) shows that forced surface waves with nonzero $\partial_{t} \mathbf{u}^{S}$ are a source of oceanic momentum and kinetic energy.

Substituting $\mathbf{u}^{E}=\mathbf{u}^{L}-\mathbf{u}^{S}$ into Eq. (15) yields a formula for the volume-integrated Eulerian-mean kinetic energy $(1 / 2)\left|\mathbf{u}^{E}\right|^{2}=$ $(1 / 2)\left|\mathbf{u}^{L}\right|^{2}+(1 / 2)\left|\mathbf{u}^{S}\right|^{2}-\mathbf{u}^{L} \cdot \mathbf{u}^{S}$

$$
\frac{d}{d t} \int \frac{1}{2}\left|\mathbf{u}^{E}\right|^{2} d V=-\int \mathbf{u}^{S} \cdot \partial_{t} \mathbf{u}^{E} d V .
$$

Due to the term on the right of (16), Eulerian-mean kinetic energy is not conserved in unstratified, inviscid flow beneath steady surface waves.

\section{Large-eddy simulations beneath growing swell}

We consider the forced growth of two deep water swells with amplitudes $a=1 \mathrm{~m}$ and $a=2 \mathrm{~m}$. Both swells grow over a time scale of $T_{w}=4 \mathrm{~h}$, have 100 -m wavelength, 8 -s period, and the horizontal Stokes drift profiles

$$
u^{S}(z, t)=\underbrace{e^{2 k z} a^{2} k \sqrt{g k}}_{\substack{\text { def } \\=} u_{\mathrm{eq}}^{S}(z)}\left(1-e^{-t^{2} / 2 T_{w}^{2}}\right),
$$


where $g=9.81 \mathrm{~m} \mathrm{~s}^{-2}, k=2 \pi / 100 \mathrm{~m}^{-1}$, and $a=1,2 \mathrm{~m}$ in (17) are chosen (i) to illustrate the swell-mediated transmission of momentum below a shallow boundary layer and (ii) to illustrate how stronger and thus deeper turbulent mixing increases the effectiveness with which the swell-transmitted momentum mixes the boundary layer.

The Stokes acceleration $\partial_{t} u^{S}$ and total stress $\partial_{t} U^{S}=\int_{-h}^{0} \partial_{t} u^{S} d z$ associated with (17) are

$$
\partial_{t} u^{S}(z, t)=\frac{t e^{-t^{2} / 2 T_{w}^{2}}}{T_{w}^{2}} u_{\mathrm{eq}}^{S}(z), \quad \text { and } \quad \partial_{t} U^{S}=\frac{t e^{-t^{2} / 2 T_{w}^{2}}}{T_{w}} \underbrace{\frac{a^{2} \sqrt{g k}}{2 T_{w}}}_{\substack{\text { def } \\=}} .
$$

In (18), the equilibrium Stokes profile $u_{\mathrm{eq}}(z)$ is defined in (17), and $\tau_{w}$ scales the stress exerted on the ocean by the atmosphere via forced swell. The maximum vertically integrated momentum forcing of the boundary layer due to (17) is

$$
\begin{aligned}
\max \left(\partial_{t} U^{S}\right) & =\left.\partial_{t} U^{S}\right|_{t=T_{w}} \\
& =\frac{\tau_{w}}{\sqrt{e}} \approx\left\{\begin{array}{lll}
1.65 \times 10^{-5} \mathrm{~m}^{2} \mathrm{~s}^{-2} & \text { for } a=1 \mathrm{~m}, \\
6.61 \times 10^{-5} \mathrm{~m}^{2} \mathrm{~s}^{-2} & \text { for } a=2 \mathrm{~m},
\end{array}\right.
\end{aligned}
$$

similar to the stress exerted by 12 -h-long wind pulses with maximum speed 4 and $8 \mathrm{~m} \mathrm{~s}^{-1}$.

\section{a. Numerical methods and software}

Our large-eddy simulations solve (9)-(11), where $\mathbf{u}^{L}$ is the resolved Lagrangian-mean velocity filtered to remove scales smaller than the numerical grid scale. Spatial filtering motivates downgradient approximations for the subfilter stress tensor and diffusive flux,

$$
\mathcal{T}_{i j}=-2 \nu_{e} \sum_{i j}^{L}, \quad \text { and } \quad q_{i}=-\kappa_{e} \partial_{i} b,
$$

in terms of the filtered strain tensor $\sum_{i j}^{L}=(1 / 2)\left(\partial_{i} u_{j}^{L}+\partial_{j} u_{i}^{L}\right)$ and filtered buoyancy gradient $\partial_{i} b$, where the indices $i=(1,2$, $3)$ correspond to the Cartesian directions $(x, y, z)$. The eddy viscosity $\nu_{e}$ and eddy diffusivity $\kappa_{e}$ in (20) are modeled with the anisotropic minimum dissipation (AMD) formalism introduced by Rozema et al. (2015) and Abkar et al. (2016), refined by Verstappen (2018), and validated and described in detail for ocean-relevant scenarios by Vreugdenhil and Taylor (2018). Additional details about (20) are given in appendix A.

We solve (9)-(11) numerically with Oceananigans, a software package developed by the authors in the Julia programming language that runs on graphics processing units (GPUs) (Bezanson et al. 2012; Besard et al. 2018). The simulations in this paper use second-order finite volume spatial discretization, second-order Adams-Bashforth time discretization, a pressure

\footnotetext{
${ }^{1}$ With drag coefficient $C_{d}=10^{-3}$, air density $\rho_{a}=1.225 \mathrm{~kg} \mathrm{~m}^{-3}$, seawater density $\rho_{w}=1035 \mathrm{~kg} \mathrm{~m}^{-3}$, and ocean-side kinematic stress parameterized with $|\tau| \approx\left(\rho_{a} / \rho_{w}\right) C_{d} u_{a}^{2}$, we find $\tau=1.65 \times$ $10^{-5} \mathrm{~m}^{2} \mathrm{~s}^{-2}$ for $u_{a}=3.7 \mathrm{~m} \mathrm{~s}^{-1}$.
}

projection method to ensure $\nabla \cdot \mathbf{u}^{L}=0$, and a fast method based on the fast Fourier transform to solve the pressure Poisson equation discretized with second-order differences on a regular grid (Schumann and Sweet 1988). Oceananigans code and documentation are hosted at https://github.com/ CliMA/Oceananigans.jl.

Our simulations are performed in two rectangular domains: one "regular" size domain with dimensions $128 \mathrm{~m} \times 128 \mathrm{~m} \times$ $64 \mathrm{~m}$ in $x, y, z$, grid spacings $0.5 \mathrm{~m} \times 0.5 \mathrm{~m} \times 0.25 \mathrm{~m}$, and resolution $256^{3}$, and a second "large" domain of dimension $192 \mathrm{~m} \times 192 \mathrm{~m} \times 96 \mathrm{~m}$ with $0.75 \mathrm{~m} \times 0.75 \mathrm{~m} \times 0.25 \mathrm{~m}$ grid spacing and resolution $256 \times 256 \times 384$. The domains are horizontally periodic in $x, y$ and have rigid top and bottom boundaries, where we impose $w^{L}=0$. To absorb internal waves radiated downward from the turbulent surface boundary layer, we implement bottom sponge layers of the form

$$
F_{\phi}=e^{-(z+H) / \delta} \mu\left(\phi^{\dagger}-\phi\right),
$$

for each variable $\phi \in(u, v, w, b)$, where $H$ is the depth of the domain, $\mu=1 / 60 \mathrm{~s}^{-1}, \partial=4 \mathrm{~m}, b^{\dagger}=N^{2} z$, and $u^{\dagger}=v^{\dagger}=w^{\dagger}=0$. Each $F_{\phi}$ is added to its corresponding equation in (9) and (10).

The simulations reported in this section and the rest of this paper are listed in Table 1. Additional information, including instructions for reproducing the simulations and figures in this paper, are hosted at https://glwagner.github.io/ WaveTransmittedTurbulence.

\section{b. Generation of a weakly turbulent initial condition}

We use preliminary simulations to generate a shallow, weakly turbulent boundary layer initial condition. The spinup simulations are forced by weak cooling associated with an upward flux of buoyancy at the surface,

$$
\left.q_{z}\right|_{z=0}=5 \times 10^{-10} \mathrm{~m}^{2} \mathrm{~s}^{-3} .
$$

The spinup simulations are run for half an inertial period until $t_{\text {spin }}=\pi / f$, where $f=10^{-4} \mathrm{~s}^{-1}$ is the Coriolis parameter. The boundary layer depth at the end of the spinup is approximately $8 \mathrm{~m}$. The horizontally averaged buoyancy and velocity at $t_{\text {spin }}=\pi / f$ are shown in Fig. 2 and serve as initial conditions for subsequent simulations reported in this section, which use the same domain, resolution, and sponge layer configuration.

\section{c. Near-inertial waves, turbulence, and boundary layer deepening}

Growing swells with the increasing Stokes drift profile in (17) and equilibrium amplitudes $a=1 \mathrm{~m}$ and $a=2 \mathrm{~m}$ are imposed on the weakly turbulent end state of the spinup simulation. The simulation time is reset to $t=0$. We impose free slip conditions on the horizontal velocity components and no normal flow on the vertical velocity component at top and bottom boundaries,

$$
w^{L}=\partial_{z} u^{L}=\partial_{z} v^{L}=0, \quad \text { at } \quad z=0 \quad \text { and } \quad z=-H .
$$

The boundary conditions (23) ensure there is no source of momentum from the boundaries. We impose a no-flux condition on buoyancy at the top boundary, 
(a)

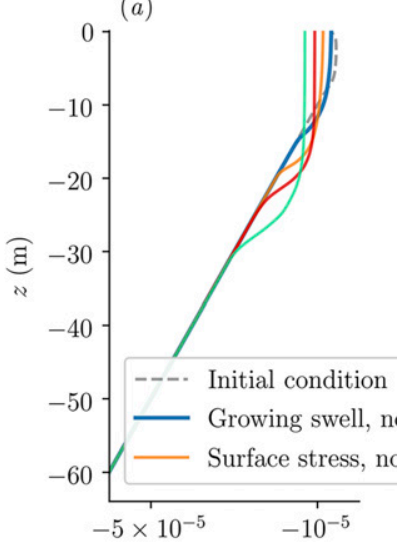

$\langle b\rangle\left(\mathrm{m} \mathrm{s}^{-2}\right)$ (b)

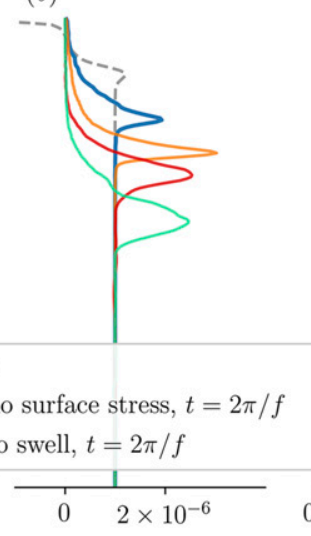

$(c)$

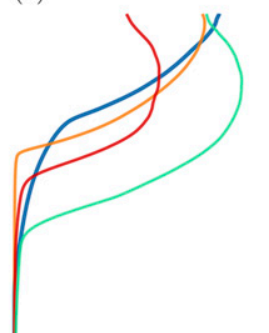

$(d)$

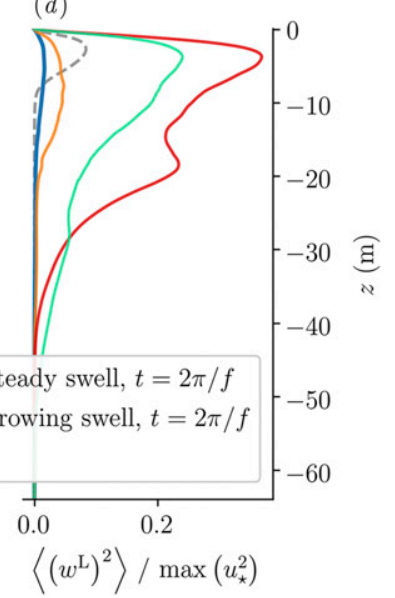

FIG. 2. Horizontally averaged fields in three LES forced by growing swell with equilibrium amplitude $a=2 \mathrm{~m}$ (solid lines), a pulse of surface stress with no waves (dashed lines) a pulse of surface stress beneath steady waves with amplitude $a=1 \mathrm{~m}$. Shown are (a) horizontally averaged buoyancy, (b) buoyancy gradient, (c) speed, and (d) vertical velocity variance normalized by $\max \left(u_{\star}^{2}\right)=1.65 \times 10^{-5} \mathrm{~m}^{2} \mathrm{~s}^{-2}$.

$$
\left.\partial_{z} b\right|_{z=0}=0 .
$$

The bottom boundary condition $\left.\partial_{z} b\right|_{z=-H}=N^{2}$ and the bottom sponge layer in (21) restore the near-bottom buoyancy profile to $N^{2} z$.

Figure 1 plots contours of vertical velocity after half an inertial period $\left.w^{L}\right|_{t=\pi / f}$ and depth profiles of the $\left\langle u^{L}\right\rangle$ and $\left\langle v^{L}\right\rangle$ for the strong swell case with equilibrium amplitude $a=2 \mathrm{~m}$. Nearinertial shear excited by the growing surface wave field drives turbulence that mixes and deepens the boundary layer. At the same time, mixing is enhanced by the organization of turbulent motions into the coherent structures of Langmuir turbulence, which manifest in the left panel of Fig. 1 as elongated rolls of alternating positive and negative vertical velocity oriented at roughly $10^{\circ}$ angles from the $y$ axis. The penetration of vertical motions through the stratified base of the boundary produces smooth downward-emanating bulbs of vertical velocity at around $z=-25 \mathrm{~m}$. Turbulent motions at the base of the boundary layer excite downward-propagating internal waves. The right panel shows the inertial rotation and downward turbulent penetration of the swell-transmitted current in time.

\section{d. Surface-concentrated versus depth-distributed stress}

To isolate the relative effect of distributed momentum forcing associated with $\partial_{t} \mathbf{u}^{S}$ in (18), we run three additional simulations for $a=1 \mathrm{~m}$ and $a=2 \mathrm{~m}$ each with the boundary condition

$$
\left.\mathcal{T}_{x z}\right|_{z=0}=-\partial_{t} U^{S}=-\tau_{w} \frac{t e^{-t^{2} / 2 T_{w}^{2}}}{T_{w}},
$$

where $\tau_{w}$ is defined in (18). The boundary condition (25) prescribes a 4-h pulse of surface stress. We combine (25) with the Stokes drift profiles

1) $u^{S}=0$ (surface stress, no swell), and

2) $u^{S}=u_{\mathrm{eq}}^{S}(z)$ for $u_{\mathrm{eq}}^{S}(z)$ in (17) (surface stress, steady swell).
For $a=1 \mathrm{~m}$ we also run a simulation forced by both the surface stress in (25) and growing swell with $\mathbf{u}^{S}(z, t)$ from (17), which we call "surface stress, growing swell." The cases described in section $3 \mathrm{c}$ are called the "growing swell, no surface stress" cases.

Figure 2 compares horizontally averaged profiles of buoyancy, buoyancy gradient, horizontal speed $\sqrt{\left\langle u^{L}\right\rangle^{2}+\left\langle v^{L}\right\rangle^{2}}$, and vertical velocity variance $\left\langle\left(w^{L}\right)^{2}\right\rangle$ after one inertial period at $t=2 \pi / f$ in the four simulations that correspond to an equilibrium wave amplitude of $a=1 \mathrm{~m}$. The maximum value of the friction velocity,

$$
\max \left(u_{\star}\right) \stackrel{\text { def }}{=} \sqrt{\max \left(\partial_{t} U^{S}\right)} \approx 4 \mathrm{~mm} \mathrm{~s}^{-1} \quad(\text { for } \quad a=1 \mathrm{~m})
$$

is used to normalize speed and vertical velocity variance. Turbulent mixing produced by growing swell deepens the boundary layer from $z \approx-8 \mathrm{~m}$ at $t=0$ to $z \approx-12 \mathrm{~m}$ at $t=2 \pi / f$. A substantial fraction of the total momentum is transmitted to laminar, nonturbulent near-inertial motions below $z \approx-12$, whose shear does not contribute to turbulent mixing. The fingerprint of momentum transmitted by swell below the boundary layer is the blue exponential tail in Fig. $2 \mathrm{c}$ below $z \approx-12 \mathrm{~m}$. The boundary layers driven by surface stress are deeper, therefore, because more of the mean kinetic energy transmitted to the boundary layer is converted to turbulence.

Figure $2 \mathrm{~d}$ shows the average vertical velocity variance $\left\langle\left(w^{L}\right)^{2}\right\rangle$. The case forced by surface stress and beneath steady swell has the strongest vertical velocities. We hypothesize this is due to the formation of vigorous Langmuir structures beneath strong, steady swell in the presence of surface stress. Vertical velocities are somewhat weaker in the case with both surface stress and growing swell, perhaps because the Stokes drift is not as strong during active momentum forcing by surface stress. Nevertheless, the surface stress, growing swell case-which experiences the strongest horizontal momentum forcing by both surface stress and swell-transmitted 
(a)

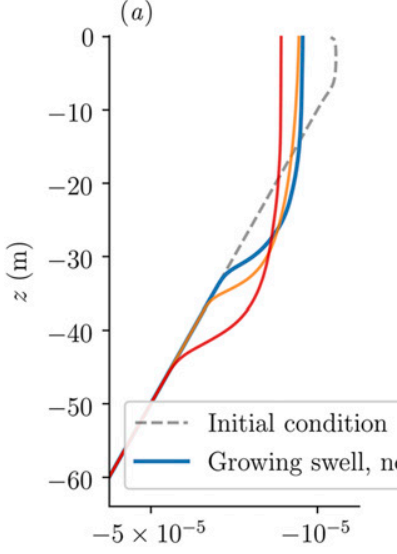

$\langle b\rangle\left(\mathrm{m} \mathrm{s}^{-2}\right)$ (b)

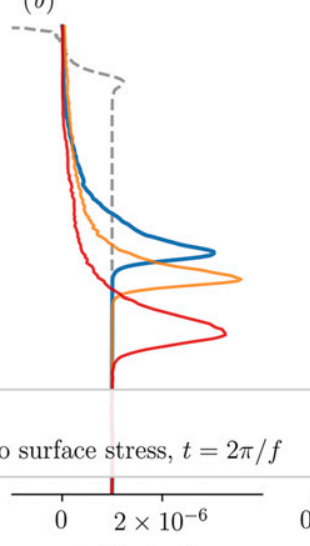

$-\frac{10}{10}$

$\partial_{z}\langle b\rangle\left(\mathrm{s}^{-2}\right)$ $(c)$

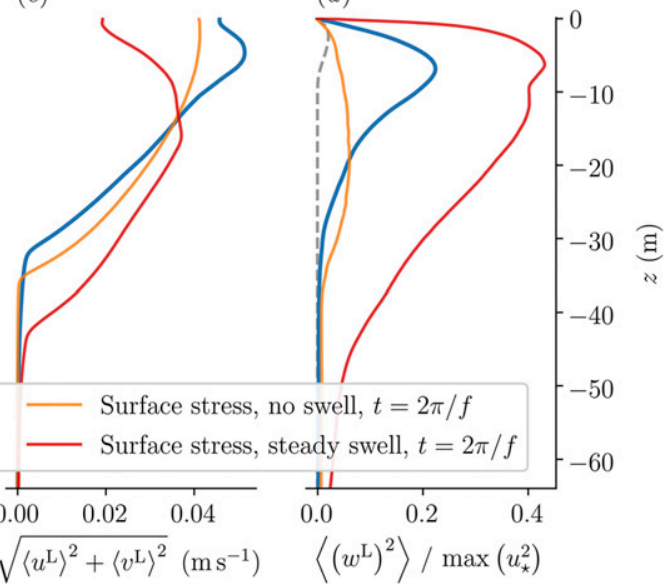

FIG. 3. Horizontally averaged fields in three LES forced by growing swell with equilibrium amplitude $a=2 \mathrm{~m}$ (solid lines), a pulse of surface stress with no waves (dashed lines) a pulse of surface stress beneath steady waves with amplitude $a=2 \mathrm{~m}$. Shown are (a) horizontally averaged buoyancy, (b) buoyancy gradient, (c) speed, and (d) vertical velocity variance normalized by $\max \left(u_{\star}^{2}\right)=6.61 \times 10^{-5} \mathrm{~m}^{2} \mathrm{~s}^{-2}$.

stress-boasts the deepest boundary layer and the strongest horizontal velocities.

Figure 3 is similar to Fig. 2 but for equilibrium wave amplitude $a=2 \mathrm{~m}$. The maximum value of the friction velocity for $a=2 \mathrm{~m}$ is

$$
\max \left(u_{\star}\right)=\sqrt{\max \left(\partial_{t} U^{S}\right)} \approx 8 \mathrm{~mm} \mathrm{~s}^{-1} .
$$

The crucial difference between the results depicted in Fig. 2 and the results in Fig. 3 is that turbulence in the growing swell simulation mixes the boundary layer to $z \approx-32 \mathrm{~m}$. This greater deepening enables greater turbulence production by shear transmitted by the growing swell, and thus mixing rates more comparable to the boundary layers driven by surface stress. The dramatic differences in vertical velocity variance between the blue lines in Figs. $2 d$ and $3 d$ are evidence that Langmuir turbulence is more active and effective in the growing swell simulation with $a=2 \mathrm{~m}$ than in the growing swell simulation with $a=1 \mathrm{~m}$. Despite strong vertical velocities beneath growing swell (blue line, Fig. 3d), which suggest the presence of Langmuir turbulence (McWilliams et al. 1997; Sullivan and McWilliams 2010), the boundary layer is deeper in the surface stress case with no swell (blue and orange lines, Fig. 3b).

Figure 4 illustrates the temporal evolution of the horizontally averaged $x$-velocity $\left\langle u^{L}\right\rangle(z, t)$ and the horizontally averaged turbulent kinetic energy

$$
E(z, t) \stackrel{\text { def }}{=}\left\langle\frac{1}{2}\left|\mathbf{u}^{L}-\left\langle\mathbf{u}^{L}\right\rangle\right|^{2}\right\rangle .
$$

The total stress exerted on the boundary layer for the "growing waves" and "surface stress, steady waves" simulations, which are plotted in the top right and left panels of Fig. 4, are identical. Yet because surface stress is concentrated at the top of the domain, it drives faster, more strongly sheared currents that transfer more of their energy to turbulent kinetic energy.
The turbulent kinetic energy with surface stress and steady wave overhead is roughly 3 times larger than with growing waves and no surface stress. The temporal structure is also different: a burst of turbulence appears beneath growing waves around $t=8 \mathrm{~h}$, while a turbulent layer steadily penetrates the stratified fluid below the surface stress over the 8 -h period of significant forcing.

\section{e. Alignment of freely decaying coherent Langmuir turbulence structures perpendicular to Stokes drift}

Figure 5 plots a time series of normalized vertical velocity contours at $z=-2 \mathrm{~m}$ and $z=-8 \mathrm{~m}$ for the surface stress, steady waves case with $a=2 \mathrm{~m}$. A black arrow in the center of each plot indicates the direction of the mean horizontal flow at each time and depth. The time series shows that the coherent Langmuir turbulence structures rotate and grow in size over time, especially after the forcing dies out after $t \approx(3 / 4) \times 2 \pi / f$.

The rotation of the coherent Langmuir turbulence structures appears fixed to the mean flow direction at early times up to $t=$ $(1 / 2) \times 2 \pi / f$, when momentum forcing is significant. After $t=$ $(1 / 2) \times 2 \pi / f$, and as the mean shear weakens, the cell appears to rotate into alignment with $\hat{\mathbf{y}}$ at around $t=2 \pi / f$. Parameter $\hat{\mathbf{y}}$ is the axis of the background vorticity associated with the surface waves,

$$
-\nabla \times \mathbf{u}^{S}=\partial_{z} u^{S} \hat{\mathbf{y}}
$$

Thus, the coherent structures align with the direction of mean shear at early times and during active forcing, as in Sullivan et al. (2012), but rotate onto the axis of the surface wave pseudovorticity at later times, during free decay.

\section{Turbulent mixing following rapid and gradual surface wave growth}

In this section we reveal some differences between the turbulent evolution of boundary layers forced by the (i) rapid and 

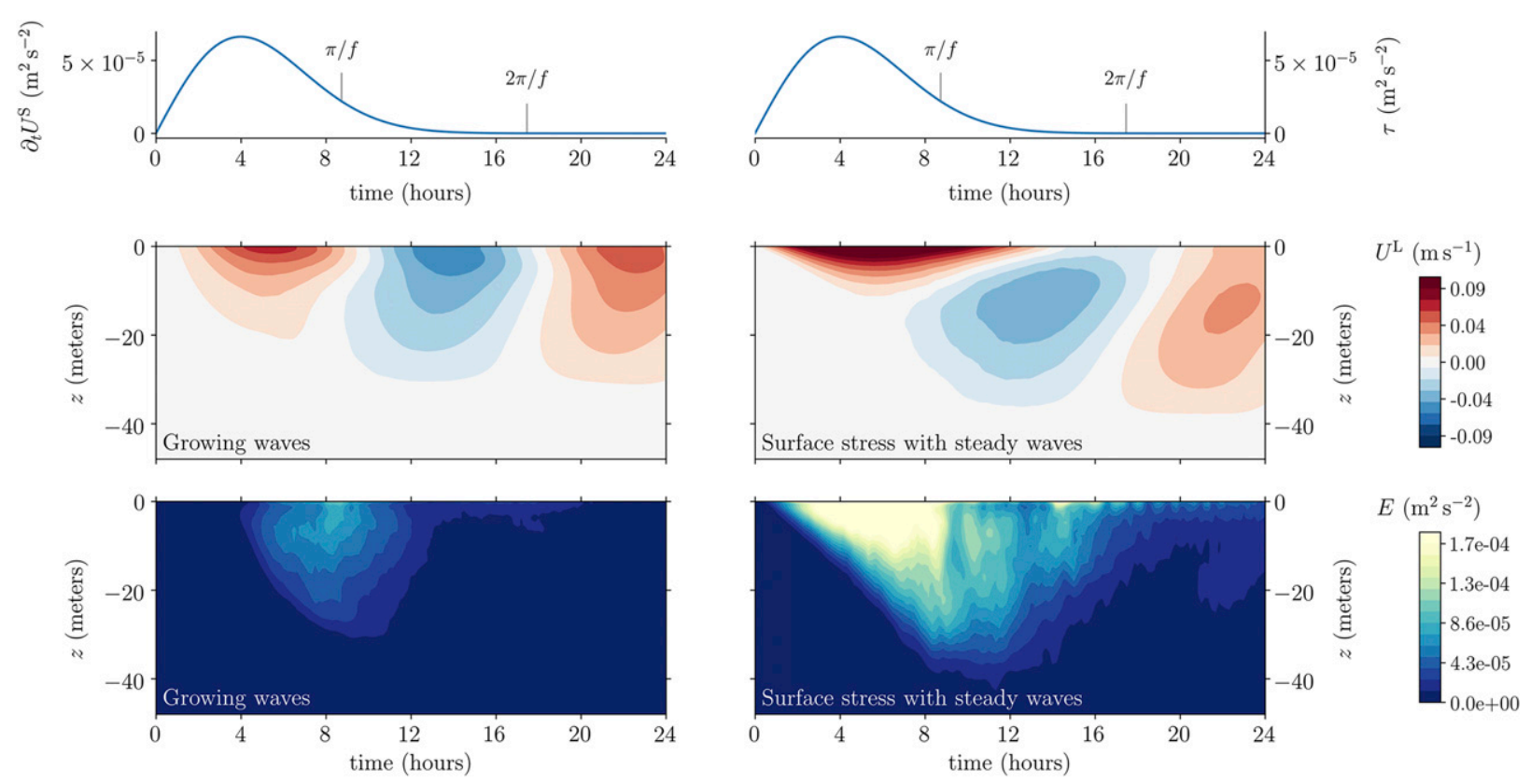

FIG. 4. A depth-time plot of the horizontally averaged $x$-velocity, $\left\langle u^{L}\right\rangle(z, t)$, and turbulent kinetic energy $E(z, t)$ defined in $(28)$.

(ii) gradual growth of a surface wave field. In short, when $f \neq 0$, the rapid growth of a surface wave field leads to the momentum distribution $\mathbf{u}^{L}=\mathbf{u}^{S}$ and thus $\mathbf{u}^{E}=0$, while the gradual growth of a surface wave field leads to $\mathbf{u}^{L}=0$. We explore the difference between these two initial conditions on turbulent mixing driven by surface stress using large-eddy simulations.

\section{a. Rapid and gradual surface wave growth over laminar boundary layers}

If $\tau$ is a prescribed function of time, the solution to (7) is

$$
U^{L}(t)=U^{L}(0)+e^{-i f t} \int_{0}^{t} e^{i f t^{\prime}}\left(\tau+\partial_{t^{\prime}} U^{S}\right) d t^{\prime}
$$

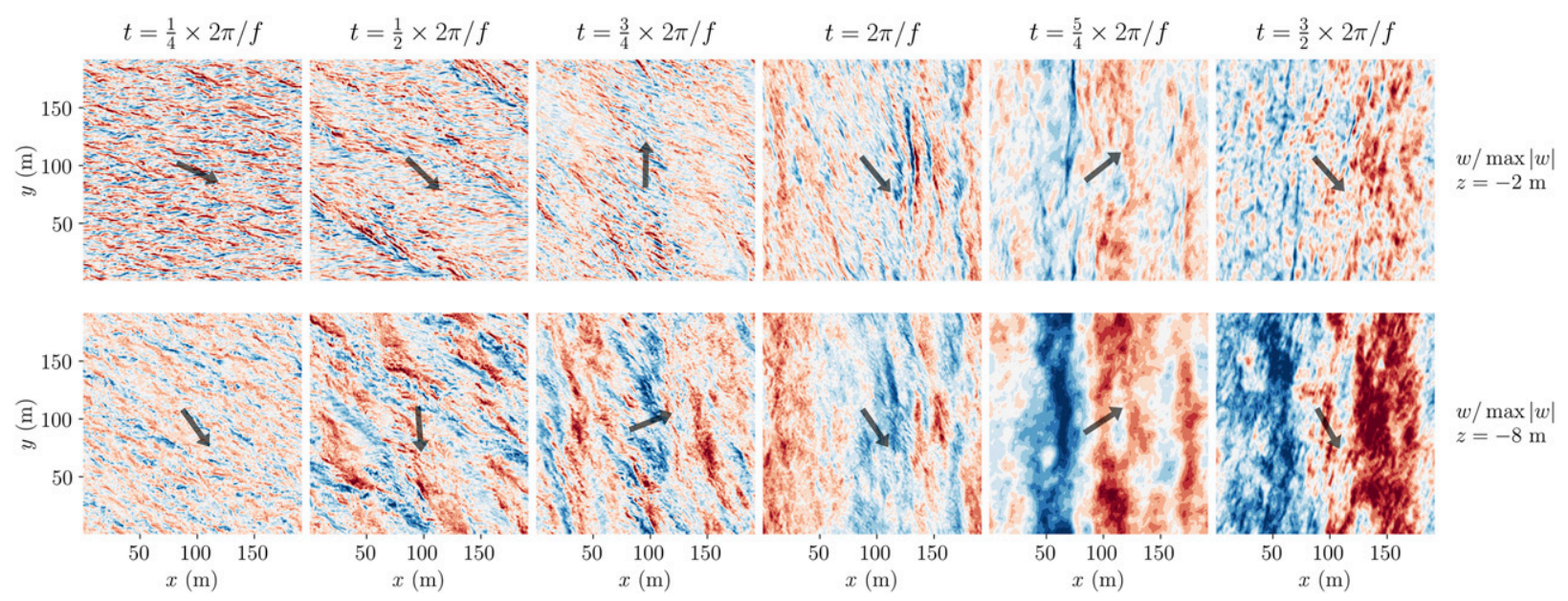

FIG. 5. Contours of vertical velocity normalized by its maximum absolute value at $z=-2 \mathrm{~m}$ and $z=-8 \mathrm{~m}$ from the strong "surface stress with steady waves" simulation (simulation 8 in Table 1), showing the rotation of coherent Langmuir turbulence structures into $\hat{\mathbf{y}}$. A thick arrow in the middle of the domain indicates the direction of the horizontally averaged horizontal flow at the same depth as the vertical velocity. The simulation is actively forced for $t \approx 12 \mathrm{~h}$; the flow begins to decay freely after $t \approx(3 / 4) \times 2 \pi / f$. At early times, the coherent structures are roughly aligned with the direction of mean shear, which is aligned with $\hat{\mathbf{x}}$, the direction of forcing. As the current rotates inertially, so do the coherent structures, though after $t \approx \pi / f$ the rotation rate of the structures begins to slow until $t \approx 2 \pi / f$, at which point the structure axes are fixed to $\hat{\mathbf{y}}$. Between $t=(5 / 4) \times 2 \pi / f$ and $t=(3 / 2) \times 2 \pi / f$, the mean shear rotates through $\hat{\mathbf{x}}$, perpendicular to the orientation of the coherent structures, which destroys much of the structures' coherence (not shown). The aftermath of this shearing event is apparent from comparing the vertical velocity at $z=-2 \mathrm{~m}$ : much of the coherency evident at $t=(5 / 4) \times 2 \pi / f$ is destroyed at $t=(3 / 2) \times$ $2 \pi / f$. The strength and coherency of the Langmuir turbulence structures thus pulsates as the near-inertial current rotates, strengthening when the current is aligned or antialigned with $\hat{\mathbf{y}}$, and shearing and disintegrating when the mean current is perpendicular to $\hat{\mathbf{y}}$. 
In the limit that surface waves grow rapidly with $\tau=0$, such that $\partial_{t} U^{S}=U_{\mathrm{eq}}^{S} \delta(t)$ for some equilibrium Stokes transport $U_{\mathrm{eq}}^{S}$, (30) is an inertial oscillation,

$$
U^{L}(t)=U^{L}(0)+e^{-i f t} U_{\mathrm{eq}}^{S}
$$

If surface waves grow over much longer time scales than $1 / f$, on the other hand, then $U^{L} \approx 0$.

A generalization of the vertically integrated result in (31) occurs beneath surface waves with the time-dependent Stokes drift field

$$
\mathbf{u}^{S}(z, t)=\mathbf{u}_{\mathrm{eq}}^{S}(z) H(t),
$$

where $H(t)$ is the Heaviside function and $\mathbf{u}_{\mathrm{eq}}^{S}(z)$ is the equilibrium Stokes drift. In this case, the Stokes drift tendency is $\partial_{t} \mathbf{u}^{S}=\mathbf{u}_{\mathrm{eq}}^{S} \delta(t)$ and Lagrangian-mean velocity field just after $t=$ 0 , when the surface wave Stokes drift profile is steady, is

$$
\lim _{t \rightarrow 0^{+}} \mathbf{u}^{L}=\mathbf{u}_{\mathrm{eq}}^{S}(z) .
$$

This flow has zero Eulerian-mean current, and corresponds to an ocean boundary layer forced by the rapid growth of a surface wave field. If stable, the initial condition (33) develops into the perfect inertial oscillation

$$
u^{L}(z, t)+i v^{L}(z, t)=e^{-i f t} u_{\mathrm{eq}}^{S}(z),
$$

with constant mean kinetic energy

$$
\frac{1}{2}\left|\mathbf{u}^{L}\right|^{2}=\frac{1}{2}\left(u_{\mathrm{eq}}^{S}\right)^{2},
$$

and oscillatory Eulerian-mean kinetic energy

$$
\frac{1}{2}\left|\mathbf{u}^{E}\right|^{2}=(1-\cos f t)\left(u_{\mathrm{eq}}^{S}\right)^{2} .
$$

In the absence of sources of vorticity such as rotation, stratification, or viscous stresses, the flow $\mathbf{u}^{E}=0$ is stable and cannot transition to turbulence. ${ }^{2}$ In stratified surface-waveaveraged flows, however, stability is guaranteed only when the Lagrangian-mean Richardson number is greater than $1 / 4$ (Holm 1996),

$$
\mathrm{Ri}^{L} \stackrel{\text { def }}{=} \frac{\partial_{z} b}{\left(\partial_{z} u^{L}\right)^{2}+\left(\partial_{z} v^{L}\right)^{2}}>\frac{1}{4} .
$$

\section{b. Large-eddy simulations of turbulent mixing driven by surface stress following rapid and gradual surface wave growth}

We use large-eddy simulations to analyze the turbulent mixing of the ocean surface boundary layer following either

\footnotetext{
${ }^{2}$ While the wave-averaged momentum is $\mathbf{u}^{L}$, the wave-averaged vorticity is $\nabla \times \mathbf{u}^{E}$ [see Bühler (2014) or chapter 2.4 in Wagner (2016)]. Thus, without a source of vorticity, a flow with $\mathbf{u}^{E}=0$ is irrotational and cannot transition to turbulence.
}

rapid surface wave growth leading to (33), or gradual surface wave growth leading to

$$
\left.\mathbf{u}^{L}\right|_{t=0}=0
$$

We refer to (33) as the "excited" state, and (38) as the "resting" state.

We choose parameters such that our results resemble those of McWilliams et al. (1997). McWilliams et al. (1997) uses the Stokes drift

$$
\mathbf{u}^{S}(z)=e^{2 k z} a^{2} k \sqrt{g k} \hat{\mathbf{x}},
$$

corresponding to a monochromatic deep water wave with amplitude $a=0.8 \mathrm{~m}$ and wavenumber $k=0.105 \mathrm{~m}^{-1}$. The surface stress and surface buoyancy flux are

$$
\begin{aligned}
\left.\mathcal{T}_{x z}\right|_{z=0} & =3.72 \times 10^{-5} \mathrm{~m}^{2} \mathrm{~s}^{-2}, \\
\left.q_{z}\right|_{z=0} & =-2.31 \times 10^{-8} \mathrm{~m}^{2} \mathrm{~s}^{-3} .
\end{aligned}
$$

Turbulent mixing is mostly driven by the surface stress in (40). The buoyancy flux in (41), which has a negligible impact on overall turbulent mixing, serves mainly to counteract the spurious laminarization of the near-surface velocity field in McWilliams et al. (1997). Our initial buoyancy profile is

$$
\left.b\right|_{t=0}=N^{2} z, \quad \text { with } \quad N^{2}=1.94 \times 10^{-5} \mathrm{~s}^{-2},
$$

superposed with small random noise. With $\partial_{\mathrm{z}} b=N^{2}$ in (42) and $\partial_{z} u^{S}=2 e^{2 k z}(a k)^{2} \sqrt{g k}, a=0.8 \mathrm{~m}, k=0.105 \mathrm{~m}^{-1}$, and $g=$ $9.81 \mathrm{~m} \mathrm{~s}^{-2}, \mathrm{Ri}^{L} \approx 0.09$ at $t=0$ and $z=0$ such that the sufficient condition for instability in (37) is met. The simulations are performed in a rectangular domain with dimensions $128 \mathrm{~m} \times$ $128 \mathrm{~m} \times 64 \mathrm{~m}$, grid spacing $0.5 \mathrm{~m} \times 0.5 \mathrm{~m} \times 0.25 \mathrm{~m}$, resolution $256^{3}$, and with sponge layers of the form (21).

We refer to cases with $a=0.8 \mathrm{~m}$ in (39) as " $1 \times$ " cases, and run two additional cases with excited and resting initial conditions and the same boundary conditions, except with 4 times stronger Stokes drift fields

$$
\mathbf{u}_{4 \times}^{S}=4 \mathbf{u}^{S},
$$

corresponding to surface waves with twice the amplitude. We finally run a "reference" simulation with $\mathbf{u}^{S}=0$. These five simulations are labeled A-E in Table 1 . A visualization of vertical velocity in the $x-y$ plane after two inertial periods such that $t=2 \times 2 \pi / f$, at a depth of $z=-4 \mathrm{~m}$, and for the reference case, $1 \times$ excited case, and $1 \times$ resting case, are shown in Fig. 6. Both the $1 \times$ excited and $1 \times$ resting cases exhibit the coherent structures of well-developed Langmuir turbulence (Sullivan and McWilliams 2010).

The main result of this section is that the excited initial conditions in (33) provide a reservoir of near-inertial shear that quickly transitions to turbulence and rapidly deepens the boundary layer at early times. This rapid early deepening, compared to the more gradual evolution of boundary layers initialized with the resting state (38), is illustrated in Figs. 7 and 8 , which depict the horizontally averaged buoyancy, vertical buoyancy gradient, and Lagrangian-mean velocity fields $\left\langle u^{L}\right\rangle$ 

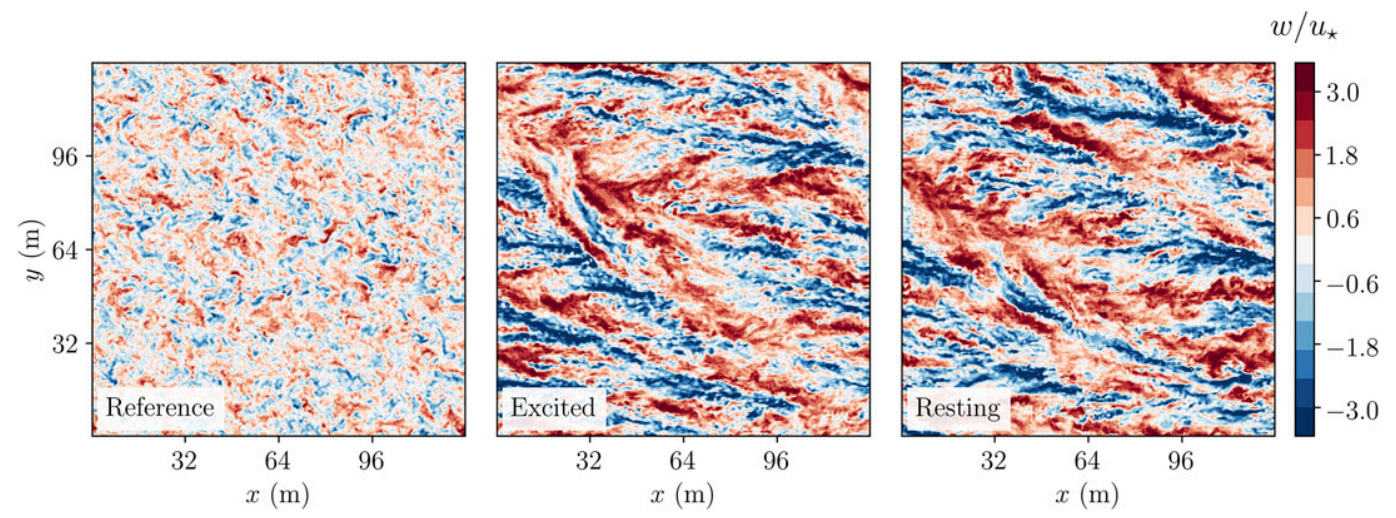

FIG. 6. Contours of vertical velocity in the $x-y$ plane and at a depth $z=-4 \mathrm{~m}$ in the reference, $1 \times$ excited, and $1 \times$ resting large-eddy simulations. Simulation parameters are detailed in Table 1.

and $\left\langle v^{L}\right\rangle$ after a quarter of an inertial period [blue lines, $t=$ $(1 / 4) \times 2 \pi / f]$ and after two inertial periods (orange lines, $t=$ $2 \times 2 \pi / f)$ in the $1 \times$ and $4 \times$ cases, respectively. The effect of $1 \times$ excited initial conditions is modest - the boundary layer is approximately $20 \%$ deeper than the $1 \times$ resting case at $t=$ $(1 / 4) \times 2 \pi / f$ - and the two boundary layer depths become similar by $t=2 \times 2 \pi / f$ as boundary layer deepening slows.

Because the surface wave field is stronger in the $4 \times$ cases, the effect of initial enhanced shear in the excited case is more dramatic: after $1 / 4$ of an inertial period, the boundary layer is almost twice as deep in the excited simulation as in the resting and reference simulations. The imprint of the initial excited state is still evident even at $t=2 \times 2 \pi / f$, at which point the excited boundary layer is still deeper than the boundary layer spun up from a resting state. We conclude with the visualization of the evolution of vertical velocity in Fig. 9 from the $4 \times$ resting and excited experiments at the early time $t=(1 / 4) \times 2 \pi / f$. In the $4 \times$ excited simulation, turbulence penetrates deeper and regions of organized vertical velocity are stronger, reflecting both the greater energy available for turbulent mixing in the excited cases, and the organization of the more energetic turbulence into stronger coherent structures.

\section{Discussion}

The simulation illustrated by Fig. 1 shows that the growth of surface waves can excite sheared near-inertial waves and drive turbulence and mixing that deepens the ocean surface boundary layer. The simulations in section 3 show that, more generally, the partitioning of ocean momentum forcing into a (a)

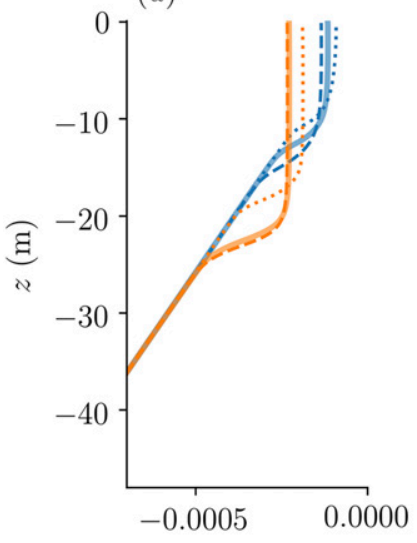

$\langle b\rangle\left(\mathrm{m} \mathrm{s}^{-2}\right)$ (b)

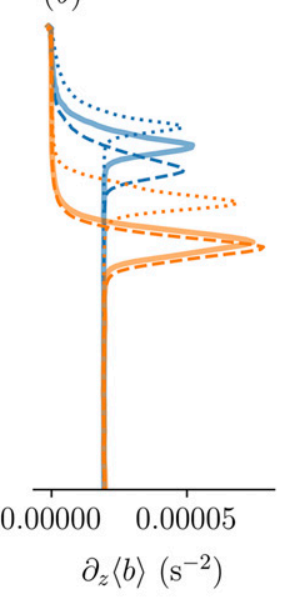

(c)

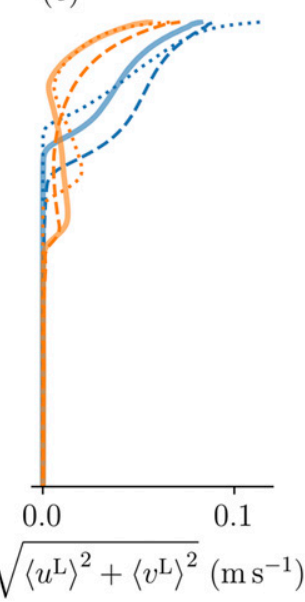

(d)

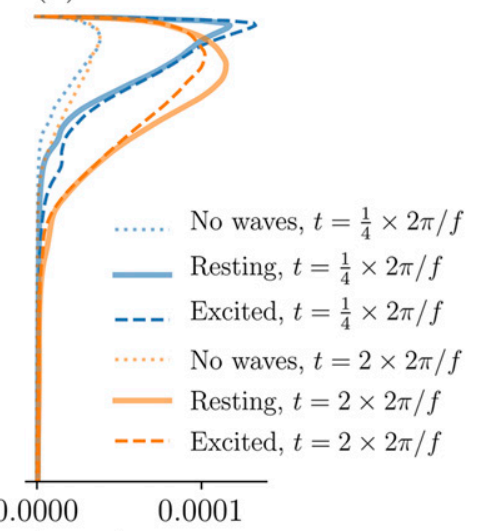

$\left\langle\left(w^{\mathrm{L}}\right)^{2}\right\rangle\left(\mathrm{m}^{2} \mathrm{~s}^{-2}\right)$

FIG. 7. Horizontally averaged fields in $1 \times$ large-eddy simulations: (a) buoyancy, (b) buoyancy gradient, (c) speed, and (d) vertical velocity variance. Excited initial conditions (dashed lines) cause an initial mixing event to deepen the boundary layer compared to resting initial conditions (solid lines). This is most evident in the buoyancy gradient profiles at $t=(1 / 4) \times 2 \pi / f$ [blue lines in (b)]. By $t=2 \times 2 \pi / f$, memory of the initial condition is lost and the buoyancy, buoyancy gradient, and vertical velocity variance profiles are similar between the excited and resting case [orange dashed and solid lines in (a), (b), and (d)]. The horizontal velocities in the excited case still exhibit faster speeds [orange dashed and solid lines in (c)] due to the excitation of a strong inertial oscillation at $t=0$. The differences between the "no waves" and "excited" cases are discussed in McWilliams et al. (1997). Figure A1 reproduces some of the plots from McWilliams et al. (1997). Simulation parameters are detailed in Table 1. 
(a)

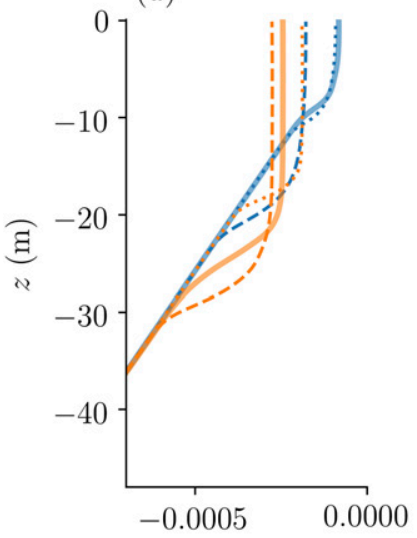

$\langle b\rangle\left(\mathrm{ms}^{-2}\right)$ $(b)$

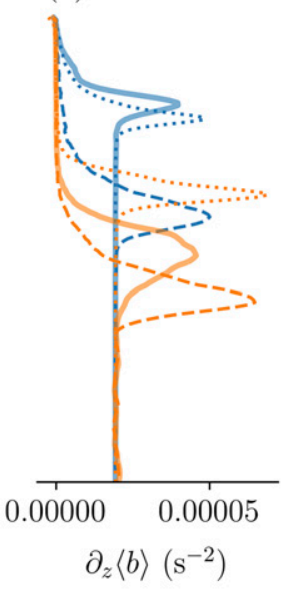

$(c)$

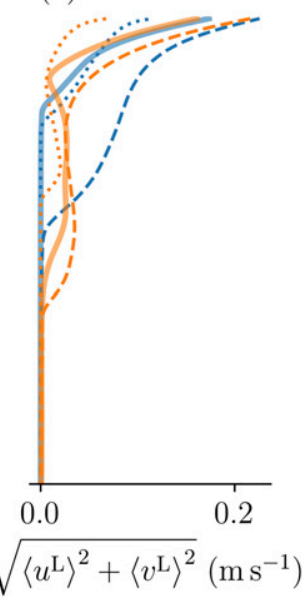

$(d)$

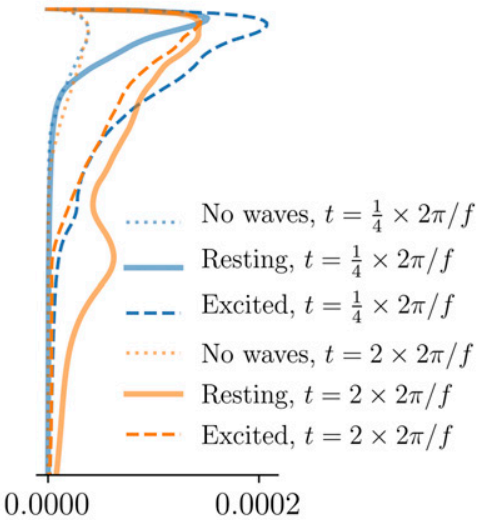

$\left\langle\left(w^{\mathrm{L}}\right)^{2}\right\rangle\left(\mathrm{m}^{2} \mathrm{~s}^{-2}\right)$

FIG. 8. As in Fig. 7, but for $4 \times$ large-eddy simulations with $4 \times$ stronger wave fields and excited initial conditions. The difference between the boundary layer depth at $t=(1 / 4) \times 2 \pi / f$ for the resting and excited cases is dramatic, as evidenced by the blue dashed and solid lines in (b). Unlike Fig. 7, memory of the initial mixing event persists at $t=2 \times 2 \pi / f$. The resting case exhibits stronger vertical velocities than the excited case at $t=2 \times 2 \pi / f$, especially at the base of the boundary layer. The reason for this is unknown.

surface-concentrated component and a depth-distributed component mediated by growing swell impacts the ensuing mean currents and turbulent evolution and deepening of the ocean surface boundary layer. The simulations in section 4 show that the rate at which swell grows also affects the evolution of the boundary layer, a fact that is well appreciated in the context of surface-concentrated momentum forcing, but less so for swell-mediated momentum forcing.

The distinction between momentum transfer via equilibrium range waves and viscous stress near or at the surface, and depth-distributed momentum transfer via the growth of swell may be important when $\partial_{t} U^{S}$ comprises a significant fraction of the total water-side stress $\tau+\partial_{t} U^{S}$. For example, Fan et al. (2009) find that $\partial_{t} U^{S}$ rises to $25 \%$ of the total stress in strongly forced hurricane conditions. However, depending on the quantity of interest, the differences between boundary layers driven by $\tau$ and boundary layers driven by $\partial_{t} U^{S}$ are probably small in typical scenarios when $\partial_{t} U^{S}$ is small compared to $\tau$.
This is especially true in boundary layers deeper than the longest swell components, where preexisting turbulence capably converts swell-deposited kinetic energy to turbulent kinetic energy.

The unimportance of the depth dependence of atmospheric momentum forcing under typical conditions has implications for parameterizations of atmospheric momentum forcing in general circulation models. Current general circulation models do not partition atmospheric momentum forcing into the two components in (7). Instead, general circulation models impose atmospheric momentum forcing through a surface stress. This approximation, which is sensible because $90 \%-95 \%$ of the stress exerted on the ocean by the atmosphere enters via equilibrium range waves with very short decay scales (Melville 1996), is further justified by our results, which suggest that the depth dependence of the remaining $5 \%-10 \%$ of the stress exerted on the ocean is relatively unimportant except in very shallow boundary layers. More realistic observations and
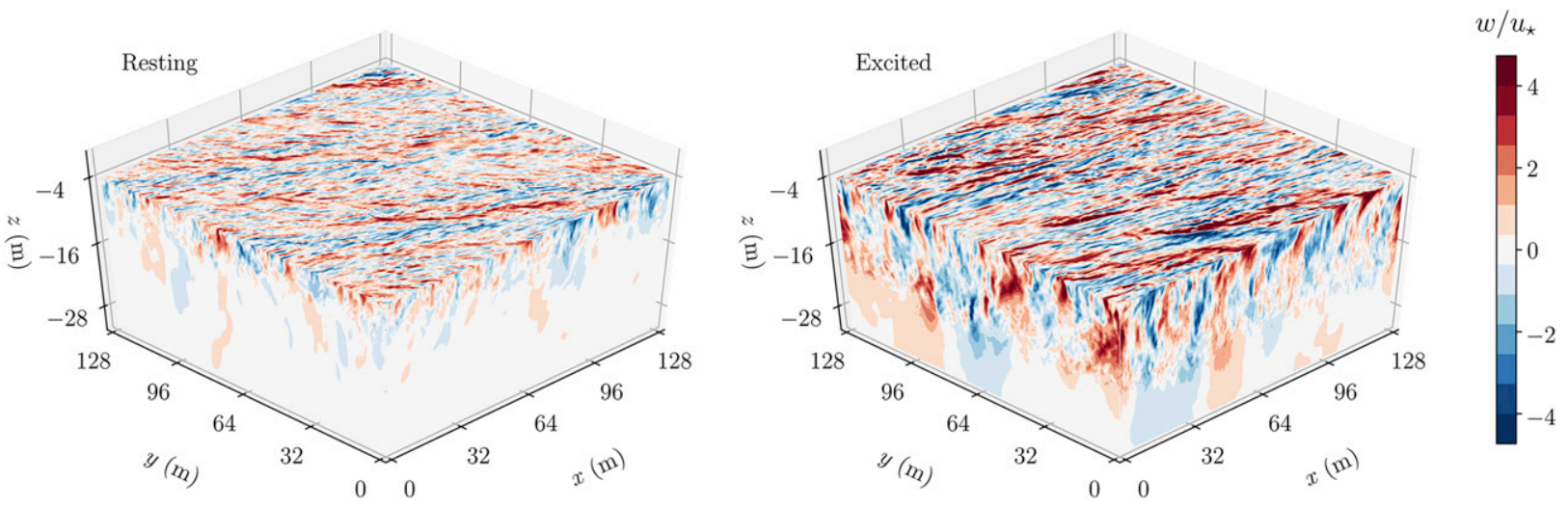

FIG. 9. Comparison of the vertical velocity at $t=(1 / 4) \times 2 \pi / f$ in two large-eddy simulations with resting and excited initial conditions. 

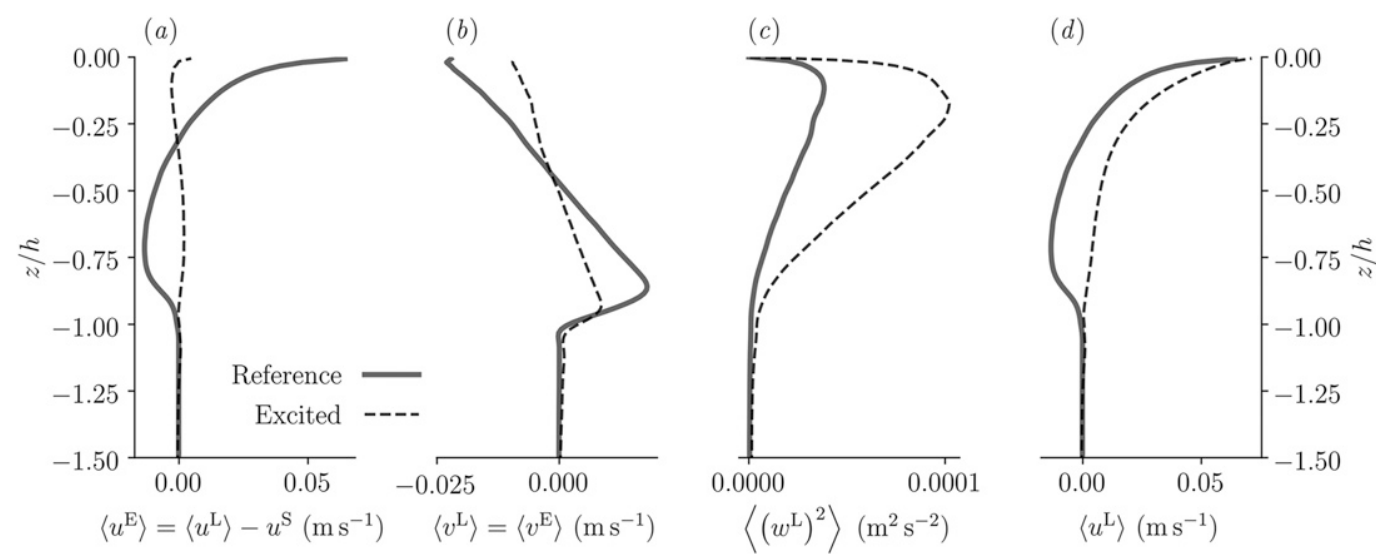

FIG. A1. (a) Horizontally averaged Eulerian-mean $x$-velocity $\left\langle u^{E}\right\rangle$, (b) horizontally averaged $y$-velocity $\left\langle v^{E}\right\rangle=\left\langle v^{L}\right\rangle$, (c) horizontally averaged vertical variance $\left\langle\left(w^{L}\right)^{2}\right\rangle$, and (d) horizontally averaged Lagrangian-mean $\left\langle u^{L}\right\rangle$. Here, $\left\langle v^{E}\right\rangle=$ $\left\langle v^{L}\right\rangle$ because the Stokes drift is in $x$, such that $\mathbf{u}^{S}=u^{S} \hat{\mathbf{x}}$. The vertical coordinate $z / h$ is height normalized by $h$, the depth of the maximum horizontally averaged buoyancy gradient such that $\partial_{z}\langle b\rangle(h)=\max \left(\partial_{z}\langle b\rangle\right)$. Panels (a) and (b) reproduce Fig. 2 in McWilliams et al. (1997). Panel (c) reproduces Fig. 6 in McWilliams et al. (1997).

modeling are warranted to further evolution of boundary layers forced by both surface stress and swell growth.

Acknowledgments. This paper's seed was planted by arguments between the lead author and Sean R. Haney about the intricacies and obscurities of Stokes drift while Sean was a postdoctoral researcher at the Scripps Institution of Oceanography. Besides being an amazing surfer and a generous friend, Sean had a unique ability to make arguments fun and productive. Sean passed away too soon due to illness on 1 January 2021 and is deeply missed. This paper is dedicated to him. Be careful, Sean.

In addition to Sean's inspiration, this work benefited from the constructive criticism of two reviewers and amiable banter with Stephen Belcher, Keaton Burns, Navid Constantinou, Baylor Fox-Kemper, Ramsey Harcourt, Qing Li, Brodie Pearson, Nick Pizzo, Brandon Reichl, and William Young. This work was supported by the generosity of Eric and Wendy Schmidt by recommendation of the Schmidt Futures program, and by the National Science Foundation under Grant AGS-6939393.

\section{APPENDIX A}

\section{Subfilter Fluxes in Wave-Averaged Large-Eddy Simulations}

Our large-eddy simulations of (9)-(11) use a downgradient hypothesis to model the subfilter stress tensor $\mathcal{T}_{i j}$ appearing in (9),

$$
\mathcal{T}_{i j}=-2\left(\nu_{e}+\nu\right) \sum_{i j}^{L}+\nu \delta_{3 j} \partial_{z} u^{S}
$$

where $\nu$ is the molecular viscosity, $\nu_{e}$ is an eddy viscosity that is a nonlinear function of resolved velocity field, $\delta_{i j}$ is the Kronecker delta, and

$$
\sum_{i j}^{L} \stackrel{\text { def }}{=} \frac{1}{2}\left(\partial_{i} u_{j}^{L}+\partial_{j} u_{i}^{L}\right)
$$

is the Lagrangian-mean rate of strain tensor. The divergence $\nabla \cdot \mathcal{T}$ in (9) is written $\partial_{j} \mathcal{T}_{i j}$ in indicial notation. The subfilter flux of buoyancy in (10) is, similarly,

$$
\mathbf{q}=-\kappa_{e} \nabla b,
$$

where $\kappa_{e}$ is the eddy diffusivity of buoyancy.

\section{a. The Lagrangian-mean strain tensor and kinetic energy dissipation}

Typical models for large-eddy simulations of (9)-(11) (Skyllingstad and Denbo 1995; Polton and Belcher 2007; Yang et al. 2015; McWilliams et al. 1997; Noh et al. 2004; Harcourt and D'Asaro 2008) use a subfilter stress tensor $\mathcal{T}_{i j}$ proportional to the Eulerian-mean rate of strain. Our subfilter model, however, uses the Lagrangian-mean rate of strain $\sum_{i j}^{L}$ in (A1). We note in advance that the following discussion above may not have significant practical import: the agreement shown in Fig. A1 between our simulations and the simulations of McWilliams et al. (1997), which use $\sum_{i j}^{E}$, suggests that the differences between models using $\sum_{i j}^{L}$ and $\sum_{i j}^{E}$ are small when resolved turbulent fluxes dominate over turbulent fluxes modeled by $\mathcal{T}_{i j}$.

The first important assumption we make is that the terms proportional to molecular viscosity $\nu$ in (A1) are negligible. This is justified after the fact by the results of our large-eddy simulations, where $\nu_{e}$ is roughly $10^{2}-10^{3}$ times larger than $\nu$ within the bulk of the boundary layer, and where $\left|\partial_{z} \mathbf{u}^{S}\right|$ is largely similar to $\left|\partial_{z} \mathbf{u}^{L}\right|$. Note that neglecting terms proportional to molecular viscosity also means we neglect the term $\nu \partial_{z} u^{S}$ in (A1) associated with the molecular dissipation of the surface wave field. This term is crucial for describing streaming flows in viscous boundary layers and other viscous surface wave phenomena at low Reynolds number (see, for example, Longuet-Higgins 1953). In this paper, we assume that molecular dissipation of the surface wave field has a negligible effect on boundary layer evolution. This assumption is almost always 
justified at the high Reynolds numbers of typical ocean surface boundary layers.

The second important assumption is that the Lagrangianmean kinetic energy, (1/2)| $\left.\mathbf{u}^{L}\right|^{2}$, undergoes a Kolmogorovian forward cascade through a spectral inertial range en route to the small, unresolved scales of molecular dissipation. The assumption of an inertial range for spectral fluxes of Lagrangianmean kinetic energy follows from the conservation of Lagrangian-mean kinetic energy in (15) in the absence of stratification, dissipation, or forcing by nonzero $\partial_{t} \mathbf{u}^{S}$. We note that an alternative hypothesis that the Eulerian-mean kinetic energy, (1/2)|u $\left.\mathbf{u}^{E}\right|^{2}$, undergoes a forward cascade through an inertial range is more difficult to justify because $(1 / 2)\left|\mathbf{u}^{E}\right|^{2}$ is not conserved [for example, Eq. (36) shows that $(1 / 2)\left|\mathbf{u}^{E}\right|^{2}$ oscillates between 0 and $2\left|\mathbf{u}^{L}\right|^{2}$ in an adiabatic inertial oscillation depending on whether $\mathbf{u}^{L}$ is aligned or antialigned with $\left.\mathbf{u}^{S}\right]$. The "turbulence-induced anti-Stokes", flow observed in Pearson's (2018) large-eddy simulations is evidence that turbulent momentum fluxes tend down Lagrangian-mean gradients, and thus tend to dissipate Lagrangian-mean kinetic energy.

Inserting $\mathcal{T}_{i j}=-2 \nu_{e} \sum_{i j}^{L}$ into (9), and maintaining $b=\partial_{\mathrm{z}} \mathbf{u}^{S}=$ 0 , we find that $\int \mathbf{u}^{L} \cdot(9) d V$ yields

$$
\frac{d}{d t} \int \frac{1}{2}\left|\mathbf{u}^{L}\right|^{2} d V=-\int 2 \nu_{e} \sum_{i j}^{L} \sum_{i j}^{L} d V .
$$

The modeled dissipation rate of Lagrangian-mean kinetic energy on the right side of (A4) is positive definite because $\nu_{e}>0$ by its definition below in (A5). As a result, $\mathcal{T}_{i j}$ in (A1) models a forward cascade of turbulent energy across the filter scale and toward molecular dissipation.

\section{b. Eddy viscosity and eddy diffusivity}

We use the anisotropic minimum dissipation model (Rozema et al. 2015; Abkar et al. 2016; Verstappen 2018) described by Vreugdenhil and Taylor (2018) for the eddy viscosity $\nu_{e}$ in (A1) and eddy diffusivity $\kappa_{e}$ in (A3). The terms $\nu_{e}$ and $\kappa_{e}$ are defined to be strictly nonnegative,

$$
\nu_{e} \stackrel{\text { def }}{=} \max \left(\nu_{e}^{\dagger}, 0\right), \quad \text { and } \quad \kappa_{e} \stackrel{\text { def }}{=} \max \left(\kappa_{e}^{\dagger}, 0\right),
$$

where the nonlinear functions $\nu_{e}^{\dagger}$ and $\kappa_{e}^{\dagger}$ are

$\nu_{e}^{\dagger} \stackrel{\text { def }}{=}-C \Delta^{2} \frac{\left(\hat{\partial}_{k} \hat{u}_{i}\right)\left(\hat{\partial}_{k} \hat{u}_{j}\right) \hat{\sum}_{i j}^{L}}{\left(\hat{\partial}_{\ell} \hat{u}_{m}\right)^{2}}$, and $\kappa^{\dagger} \stackrel{\text { def }}{=}-C \Delta^{2} \frac{\left(\hat{\partial}_{k} \hat{u}_{i}\right)\left(\hat{\partial}_{k} b\right) \hat{\partial}_{i} b}{\left(\hat{\partial}_{\ell} b\right)^{2}}$.

In (A6), $\Delta$ is the filter width defined via

$$
\frac{1}{\Delta^{2}} \stackrel{\text { def }}{=} \frac{1}{3}\left(\frac{1}{\Delta_{x}^{2}}+\frac{1}{\Delta_{y}^{2}}+\frac{1}{\Delta_{z}^{2}}\right)
$$

in terms of the potentially anisotropic $x, y, z$ grid spacings $\Delta_{i}$. The hats in (A6) denote the scaled quantities

$$
\hat{\partial}_{i} \stackrel{\text { def }}{=} \Delta_{i} \partial_{i}, \quad \hat{u}_{i} \stackrel{\text { def }}{=} \frac{u_{i}^{L}}{\Delta_{i}}, \quad \text { and } \quad \hat{\Sigma}^{L} \stackrel{\text { def }}{=} \frac{1}{2}\left(\hat{\partial}_{j} \hat{u}_{i}+\hat{\partial}_{i} \hat{u}_{j}\right)
$$

The term $C$ in (A6) is a model constant. For simulations A-E in Table 1 , which are reported in section 4 , we set $C=1 / 12$ following Verstappen (2018) and Vreugdenhil and Taylor (2018). For simulations $1-8$ in Table 1 and reported in section 3 , we implement ${ }^{3}$ a model that increases $C$ from $1 / 12$ to $2 / 3$ at $z=0$ over a scale of $4 \Delta_{z}=1 \mathrm{~m}$,

$$
C(z)=C_{I}+e^{z / d}\left(C_{0}-C_{I}\right)
$$

where $C_{I}=1 / 12, C_{0}=2 / 3$, and $d=1 \mathrm{~m}$. The model constant enhancement in (A9) is necessary for obtaining smooth buoyancy profiles near the surface during the spinup simulations 1 and 6 in Table 1 . We find that without an enhancement of the kind in (A9), the eddy diffusivity $\kappa_{e}$ is too small near $z=$ 0 during free convection, which prevents a smooth transition between the boundary-adjacent cells where the unresolved diffusive flux $\mathbf{q}$ dominates, and the turbulent interior where advective fluxes $\mathbf{u} b$ in (10) control the evolution of the buoyancy distribution.

Our use of $u_{i}^{L}$ in (A8) is justified by deriving $\nu_{e}$ and $\kappa_{e}$ from the Lagrangian-mean velocity gradient energy equation (Rozema et al. 2015). In this derivation, the term $-\left(\nabla \times \mathbf{u}^{S}\right) \times \mathbf{u}^{L}$ in (9) arises as a "transport term" in the velocity gradient energy equation, and thus, similar to Coriolis accelerations, does not affect the form of $\nu_{e}$ (Abkar et al. 2016).

\section{c. Model validation}

Figure A1 plots the horizontally averaged velocity and vertical variance from the $1 \times$ excited case, reproducing parts of Figs. 2 and 6 from McWilliams et al. (1997). Our results are similar despite the differences between our subfilter flux model and McWilliams et al.'s (1997).

\section{APPENDIX B}

\section{Lagrangian-Mean Form of the Craik-Leibovich Equations}

The Eulerian-mean form of the Craik-Leibovich momentum equation (Leibovich 1977; Suzuki and Fox-Kemper 2016) is

$$
\partial_{t} \mathbf{u}^{E}+\left(\mathbf{u}^{E} \cdot \nabla\right) \mathbf{u}^{E}-\mathbf{u}^{S} \times\left(\nabla \times \mathbf{u}^{E}\right)+f \hat{\mathbf{z}} \times \mathbf{u}^{L}+\nabla \phi=b \hat{\mathbf{z}},
$$

where

$$
\phi \stackrel{\text { def }}{=} p+\frac{1}{2}\left|\mathbf{u}^{S}\right|^{2}+\mathbf{u}^{S} \cdot \mathbf{u}^{E},
$$

$p$ is the Eulerian-mean pressure, and $\mathbf{u}^{S} \times\left(\nabla \times \mathbf{u}^{E}\right)$ is the "vortex force." Some algebraic gymnastics lead from (B1) to (9). Starting with the vector identity

$$
\begin{aligned}
\mathbf{u}^{L} \times\left(\nabla \times \mathbf{u}^{S}\right)+\mathbf{u}^{S} \times\left(\nabla \times \mathbf{u}^{L}\right)= & \nabla \cdot\left(\mathbf{u}^{S} \cdot \mathbf{u}^{L}\right)-\left(\mathbf{u}^{S} \cdot \nabla\right) \mathbf{u}^{L} \\
& -\left(\mathbf{u}^{L} \cdot \nabla\right) \mathbf{u}^{S}
\end{aligned}
$$

\footnotetext{
${ }^{3}$ See https://github.com/glwagner/WaveTransmittedTurbulence/ blob/master/src/les.jl.
} 
and subtracting both $\mathbf{u}^{S} \times\left(\nabla \times \mathbf{u}^{L}\right)=\mathbf{u}^{S} \times\left(\nabla \times \mathbf{u}^{E}\right)+\mathbf{u}^{S} \times$ $\left(\nabla \times \mathbf{u}^{S}\right)$ and

$$
\mathbf{u}^{S} \times\left(\nabla \times \mathbf{u}^{S}\right)=\nabla\left(\frac{1}{2}\left|\mathbf{u}^{S}\right|^{2}\right)-\left(\mathbf{u}^{S} \cdot \nabla\right) \mathbf{u}^{S},
$$

yields the identity

$$
\begin{aligned}
\mathbf{u}^{L} & \times\left(\nabla \times \mathbf{u}^{S}\right)+\mathbf{u}^{S} \times\left(\nabla \times \mathbf{u}^{E}\right)+\left(\mathbf{u}^{S} \cdot \nabla\right) \mathbf{u}^{E}+\left(\mathbf{u}^{L} \cdot \nabla\right) \mathbf{u}^{S} \\
& -\nabla\left(\frac{1}{2}\left|\mathbf{u}^{S}\right|^{2}+\mathbf{u}^{S} \cdot \mathbf{u}^{E}\right)=0
\end{aligned}
$$

Equation (B5) then implies that

$$
\begin{aligned}
\left(\mathbf{u}^{E} \cdot \nabla\right) \mathbf{u}^{E}-\mathbf{u}^{S} \times\left(\nabla \times \mathbf{u}^{E}\right)+\nabla \phi= & \left(\mathbf{u}^{L} \cdot \nabla\right) \mathbf{u}^{L} \\
& -\left(\nabla \times \mathbf{u}^{S}\right) \times \mathbf{u}^{L}+\nabla p
\end{aligned}
$$

where we have used (B2).

With the identity (B6), $\partial_{t} \mathbf{u}^{E}=\partial_{t} \mathbf{u}^{L}-\partial_{t} \mathbf{u}^{S}$, and the assumption $\nabla \cdot \mathbf{u}^{S}=0$ (valid for weakly modulated waves, as discussed in section 2), we can convert the Eulerian-mean form of the Craik-Leibovich equation (B1) and the continuity equation $\nabla \cdot \mathbf{u}^{E}=0$ into their Lagrangian-mean counterparts (9) and (11).

Finally, we note that (B1) is derived by Leibovich (1980) from the generalized Lagrangian-mean momentum equation presented in Theorem I of Andrews and McIntyre (1978). Thus, Leibovich (1980) provides a link between Andrews and McIntyre (1978) and our (9)-(11). As discussed by Leibovich (1980), the pseudomomentum appearing in Theorem I of Andrews and McIntyre (1978) and the surface wave Stokes drift $\mathbf{u}^{S}$ are nearly equivalent the scenarios we consider with relatively slow background rotation and slowly modulated waves.

\section{REFERENCES}

Abkar, M., H. J. Bae, and P. Moin, 2016: Minimum-dissipation scalar transport model for large-eddy simulation of turbulent flows. Phys. Rev. Fluids, 1, 041701, https://doi.org/10.1103/ PhysRevFluids.1.041701.

Andrews, D. G., and M. McIntyre, 1978: An exact theory of nonlinear waves on a Lagrangian-mean flow. J. Fluid Mech., 89, 609-646, https://doi.org/10.1017/S0022112078002773.

Besard, T., C. Foket, and B. De Sutter, 2018: Effective extensible programming: Unleashing Julia on GPUs. IEEE Trans. Parallel Distrib. Syst., 30, 827-841, https://doi.org/ 10.1109/TPDS.2018.2872064.

Bezanson, J., S. Karpinski, V. B. Shah, and A. Edelman, 2012: Julia: A fast dynamic language for technical computing. arXiv, 27 pp., https://arxiv.org/abs/1209.5145.

Bühler, O., 2014: Waves and Mean Flows. 2nd ed. Cambridge University Press, $374 \mathrm{pp}$.

Craik, A. D., and S. Leibovich, 1976: A rational model for Langmuir circulations. J. Fluid Mech., 73, 401-426, https:// doi.org/10.1017/S0022112076001420.

D’Asaro, E. A., J. Thomson, A. Shcherbina, R. Harcourt, M. Cronin, M. Hemer, and B. Fox-Kemper, 2014: Quantifying upper ocean turbulence driven by surface waves. Geophys. Res. Lett., 41, 102-107, https://doi.org/10.1002/2013GL058193.
Fan, Y., I. Ginis, and T. Hara, 2009: The effect of wind-wave-current interaction on air-sea momentum fluxes and ocean response in tropical cyclones. J. Phys. Oceanogr., 39, 1019-1034, https:// doi.org/10.1175/2008JPO4066.1.

Grare, L., W. L. Peirson, H. Branger, J. W. Walker, J.-P. Giovanangeli, and V. Makin, 2013: Growth and dissipation of wind-forced, deep-water waves. J. Fluid Mech., 722, 5-50, https://doi.org/10.1017/jfm.2013.88.

Harcourt, R. R., and E. A. D'Asaro, 2008: Large-eddy simulation of Langmuir turbulence in pure wind seas. J. Phys. Oceanogr., 38, 1542-1562, https://doi.org/10.1175/2007JPO3842.1.

Hasselmann, K., 1970: Wave-driven inertial oscillations. Geophys. Astrophys. Fluid Dyn., 1, 463-502, https://doi.org/10.1080/ 03091927009365783.

Holm, D. D., 1996: The ideal Craik-Leibovich equations. Physica D, 98, 415-441, https://doi.org/10.1016/0167-2789(96)00105-4.

Huang, N. E., 1979: On surface drift currents in the ocean. J. Fluid Mech., 91, 191-208, https://doi.org/10.1017/S0022112079000112.

Kukulka, T., A. J. Plueddemann, J. H. Trowbridge, and P. P. Sullivan, 2010: Rapid mixed layer deepening by the combination of Langmuir and shear instabilities: A case study. J. Phys. Oceanogr., 40, 2381-2400, https://doi.org/10.1175/2010JPO4403.1.

Large, W. G., E. G. Patton, A. K. DuVivier, P. P. Sullivan, and L. Romero, 2019: Similarity theory in the surface layer of large-eddy simulations of the wind-, wave-, and buoyancyforced southern ocean. J. Phys. Oceanogr., 49, 2165-2187, https://doi.org/10.1175/JPO-D-18-0066.1.

Leibovich, S., 1977: On the evolution of the system of wind drift currents and Langmuir circulations in the ocean. Part I. Theory and averaged current. J. Fluid Mech., 79, 715-743, https://doi.org/10.1017/S002211207700041X.

_- 1980: On wave-current interaction theories of Langmuir circulations. J. Fluid Mech., 99, 715-724, https://doi.org/ 10.1017/S0022112080000857.

Longuet-Higgins, M. S., 1953: Mass transport in water waves. Philos. Trans. Roy. Soc. London, 245A, 535-581, https:// doi.org/10.1098/rsta.1953.0006.

_- 1969: A nonlinear mechanism for the generation of sea waves. Proc. Roy. Soc. London, 311A, 371-389, https:// doi.org/10.1098/rspa.1969.0123.

__ 1986: Eulerian and Lagrangian aspects of surface waves. J. Fluid Mech., 173, 683-707, https://doi.org/10.1017/S0022112086001325.

Mcintyre, M., 1981: On the 'wave momentum' myth. J. Fluid Mech., 106, 331-347, https://doi.org/10.1017/S0022112081001626.

McWilliams, J. C., P. P. Sullivan, and C.-H. Moeng, 1997: Langmuir turbulence in the ocean. J. Fluid Mech., 334, 1-30, https:// doi.org/10.1017/S0022112096004375.

Melville, W. K., 1996: The role of surface-wave breaking in air-sea interaction. Annu. Rev. Fluid Mech., 28, 279-321, https:// doi.org/10.1146/annurev.fl.28.010196.001431.

Noh, Y., H. S. Min, and S. Raasch, 2004: Large-eddy simulation of the ocean mixed layer: The effects of wave breaking and Langmuir circulation. J. Phys. Oceanogr., 34, 720-735, https://doi.org/ 10.1175/1520-0485(2004)034<0720:LESOTO > 2.0.CO;2.

Pearson, B., 2018: Turbulence-induced anti-Stokes flow and the resulting limitations of large-eddy simulation. J. Phys. Oceanogr., 48, 117-122, https://doi.org/10.1175/JPO-D-17-0208.1.

Phillips, O., 1985: Spectral and statistical properties of the equilibrium range in wind-generated gravity waves. J. Fluid Mech., 156, 505-531, https://doi.org/10.1017/S0022112085002221.

Pollard, R. T., 1970: Surface waves with rotation: An exact solution. J. Geophys. Res., 75, 5895-5898, https://doi.org/ 10.1029/JC075i030p05895. 
Polton, J. A., and S. E. Belcher, 2007: Langmuir turbulence and deeply penetrating jets in an unstratified mixed layer. J. Geophys. Res., 112, C09020, https://doi.org/10.1029/2007JC004205.

Rozema, W., H. J. Bae, P. Moin, and R. Verstappen, 2015: Minimum-dissipation models for large-eddy simulation. Phys. Fluids, 27, 085107, https://doi.org/10.1063/1.4928700.

Schumann, U., and R. A. Sweet, 1988: Fast Fourier transforms for direct solution of Poisson's equation with staggered boundary conditions. J. Comput. Phys., 75, 123-137, https://doi.org/ 10.1016/0021-9991(88)90102-7.

Seshasayanan, K., and B. Gallet, 2019: Surface gravity waves propagating in a rotating frame: The Ekman-Stokes instability. Phys. Rev. Fluids, 4, 104802, https://doi.org/10.1103/PhysRevFluids. 4.104802.

Skyllingstad, E. D., and D. W. Denbo, 1995: An ocean large-eddy simulation of Langmuir circulations and convection in the surface mixed layer. J. Geophys. Res., 100, 8501-8522, https:// doi.org/10.1029/94JC03202.

Stokes, G. G., 1847: On the theory of oscillatory waves. Trans. Cambridge Philos. Soc., 8, 441-455.

Sullivan, P. P., and J. C. McWilliams, 2010: Dynamics of winds and currents coupled to surface waves. Annu. Rev. Fluid Mech., 42, 19-42, https://doi.org/10.1146/annurev-fluid-121108-145541.

, L. Romero, J. C. McWilliams, and W. K. Melville, 2012: Transient evolution of Langmuir turbulence in ocean boundary layers driven by hurricane winds and waves. J. Phys. Oceanogr., 42, 1959-1980, https://doi.org/10.1175/JPO-D-12-025.1.

Suzuki, N., and B. Fox-Kemper, 2016: Understanding Stokes forces in the wave-averaged equations. J. Geophys. Res. Oceans, 121, 3579-3596, https://doi.org/10.1002/2015JC011566.

Ursell, F., and G. Deacon, 1950: On the theoretical form of ocean swell on a rotating earth. Geophys. J. Int., 6, 1-8, https:// doi.org/10.1111/j.1365-246X.1950.tb02968.x.

Verstappen, R., 2018: How much eddy dissipation is needed to counterbalance the nonlinear production of small, unresolved scales in a large-eddy simulation of turbulence? Comput. Fluids, 176, 276-284, https://doi.org/10.1016/j.compfluid. 2016.12.016.

Vreugdenhil, C. A., and J. R. Taylor, 2018: Large-eddy simulations of stratified plane Couette flow using the anisotropic minimumdissipation model. Phys. Fluids, 30, 085104, https://oi.org/ 10.1063/1.5037039.

Wagner, G. L., 2016: On the coupled evolution of oceanic internal waves and quasi-geostrophic flow. Ph.D. thesis, University of California, San Diego, 216 pp.

Yang, D., B. Chen, M. Chamecki, and C. Meneveau, 2015: Oil plumes and dispersion in Langmuir, upper-ocean turbulence: Large-eddy simulations and K-profile parameterization. J. Geophys. Res. Oceans, 120, 4729-4759, https:// doi.org/10.1002/2014JC010542. 